Terr. Atmos. Ocean. Sci., Vol. 18, No. 2, 295-325, June 2007

\title{
Core Slabbing and Nannofossil Analysis on the Chelungpu Fault Zone, Taichung, Taiwan
}

\author{
Jong-Chang $\mathrm{Wu}^{1}{ }^{1,3}{ }^{*}$, Shiuh-Tsann Huang ${ }^{1}$, Ming-Huei Wang ${ }^{1}$, Chin-Chun Tsai ${ }^{1}$, Wen-Wei Mei ${ }^{1}$, \\ Jih-Hao Hung ${ }^{2}$, Tung- Yi Lee ${ }^{3}$, Kenn-Ming Yang ${ }^{1}$, and Kun-Fa Lee ${ }^{1,4}$
}

(Manuscript received 27 December 2005, in final form 15 January 2007)

\begin{abstract}
The results of this nannofossil analysis supply essential information for determining the formation boundaries in the upper Pliocene to Pleistocene. These results also verify the existence of a repetition fossil zone.

The TCDP well-A was sunk through the soft fine-grain muddy sandstone and mudstone dominated formations of the Pliocene and Pleistocene in the Taichung area. This study determines methods for providing core preservation in wells at fault zones and establishes a nannofossil biostratigraphy for the integrated Taiwan Chelungpu-fault Drilling Project (TCDP). Good core fabrics are useful for core description and sampling. In this present study, over $\mathbf{4 0 0}$ meters of subsurface cores were covered in resin and slabbed. Digitized images were created for all the core fabrics. More than 150 rock samples were analyzed for nannofossils to give a detailed appraisal of the biostratigraphic column of TCDP well-A.

A fossil zone at a depth interval of $431-869 \mathrm{~m}$ is a NN16 - 18 biozone. This zone is within the Cholan Formation, a lithologic stratigraphy in northern and central Taiwan. The depth interval $883-1226 \mathrm{~m}$ is NN15, and is

\footnotetext{
${ }^{1}$ Exploration and Development Research Institute, Chinese Petroleum Corporation, Miaoli, Taiwan, ROC

2 Department of Earth Sciences and Institute of Geophysics, National Central University, Chung-Li, Taiwan, ROC

${ }^{3}$ Department of Earth Science, National Taiwan Normal University, Taipei, Taiwan, ROC

4 Institute of Applied Geophysics, National Taiwan Ocean University, Keelung, Taiwan, ROC

* Corresponding author address: Dr. Jong-Chang Wu, Exploration and Development Research Institute, Chinese Petroleum Corporation, Miaoli, Taiwan, ROC; E-mail: 048704@cpc.com.tw doi: 10.3319/TAO.2007.18.2.295(TCDP)
} 
within the Chinshui Shale. The Chelungpu fault is composed of five major shear zones. These are all found at depth within the marine Chinshui Shale. At a depth interval of 1293.37 - $1710 \mathrm{~m}$ is a NN12 - 14 biozone; this interval is within the Kueichulin Formation.

Interestingly, both the interval beneath $1714 \mathrm{~m}$ and the nannofossil zone near the well bottom are NN16 - 18 (Cholan Formation), indicating a repeat of the Cholan Formation. The lowest fossil zone is also abundant in secondary reworked fossils in its assemblages. Hence, the repetition of the younger fossil zone, NN16 - 18, at the bottom of the well verifies the subsurface position of the Sanyi Fault and indicates that TCDP well-A must have passed through it.

(Key words: Nannofossil analysis, Biostratigraphy, Chelungpu fault, Taichung area)

\section{INTRODUCTION}

The Taiwan Chelungpu-fault Drilling Project (TCDP) is an integrated international study of the kinematics and dynamic behaviors of the 1999 Chi-Chi earthquake. TCDP well-A retrieved cores from $430 \mathrm{~m}$ to a depth of $2000 \mathrm{~m}$ (Figs. 1, 2). During the TCDP, preparation of the precious soft cores for different regimes became an important task. This project required lineate core preservation and abundant core sampling to satisfy the many core analyses that were to be conducted. Therefore, it was vital that all geologists communicated and cooperated well to their mutual benefit. The Exploration and Development Research Institute (EDRI) of the Chinese Petroleum Corporation (CPC) took over the task of core preservation and core slabbing using an internationally recognized standard process. It was then necessary to use low temperature storage to prevent temperature variation induced fractures in the cores. As expected, TCDP well-A passed through the Pleistocene and Pliocene half-soft rock formations of the Cholan, Chinshui Shale, and Kueichulin Formations. This fragile fault-related rock provided enhanced difficulties during core slabbing. However, the staff at the core laboratory in EDRI had some previous experience in processing this kind of half-soft rock in 2002 (Huang et al. 2002; Hung et al. 2001).

These Plio-Pleistocene cores are classified as the upper sequences of the Taichung Basin, and are dominated by very fine-grain muddy sandstones and even mudstone facies. Therefore, age verification during drilling is necessary to preserve the integrity of the nannofossil record. The staff of EDRI also took over the task of stratigraphic analysis to help in the engineering progress and assist in identification of formations during well drilling.

\section{CORE SLABBING}

The procedures followed for the half-soft core slabbing of core samples for TCDP well-A are shown in Fig. 3. The first longitudinal cores retrieved were put into a cool room for storage. While the cores were dry, resin was applied to the upper surface of each core. After about 10 days, 
resin was applied to the remaining surface of the same cores. Then, these cores were cut into two symmetric portions. If, however, advanced secondary core slabbing was requested, this slabbing process was more complicated. Before a secondary cut could be made, it was necessary to resin the fresh surface of the first cut and then wait an additional 10 days for the core to dry. Finally, these core portions were divided into three parts: portion A to be kept for longterm preservation or post completion of a detailed description be available for limited sampling; portion $\mathrm{B}$, the intermediate unit, to be permanently preserved and fixed to the bottom of a core box using epoxy resin; and portion $\mathrm{C}$ to be made available for analysis. Generally, the supply of portion $\mathrm{C}$ is not enough for most of laboratories so that it is necessary to resin the fresh core surface to protect the sample from being crushed during the slabbing process.

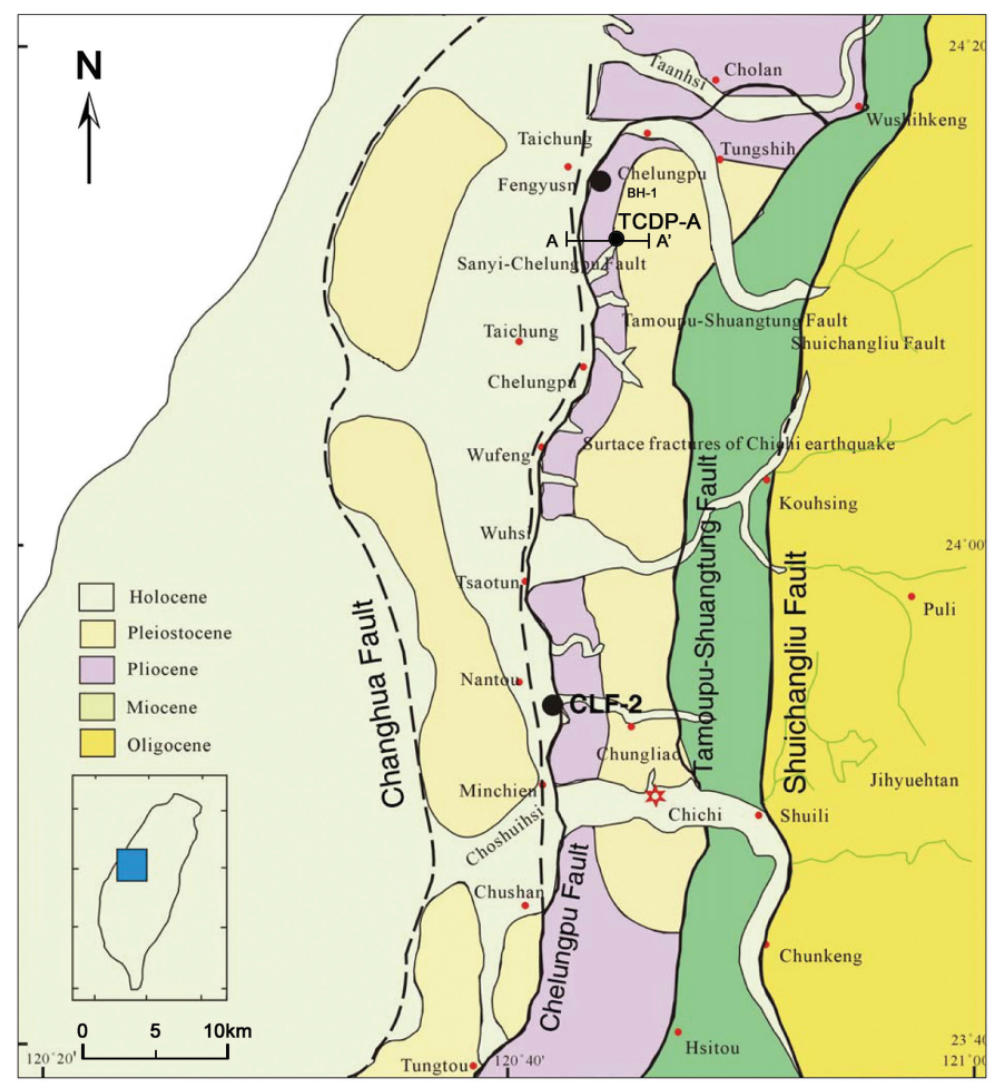

Fig. 1. Geological map of Taichung area. TCDP well-A is located at the northern part of the Chelungpu fault. The Chelungpu fault is shown by the black line while the dashed lines near the Chelungpu Fault are the previous position determined by geologists of the Chinese Petroleum Corporation before the Chi-Chi earthquake, 1999. 


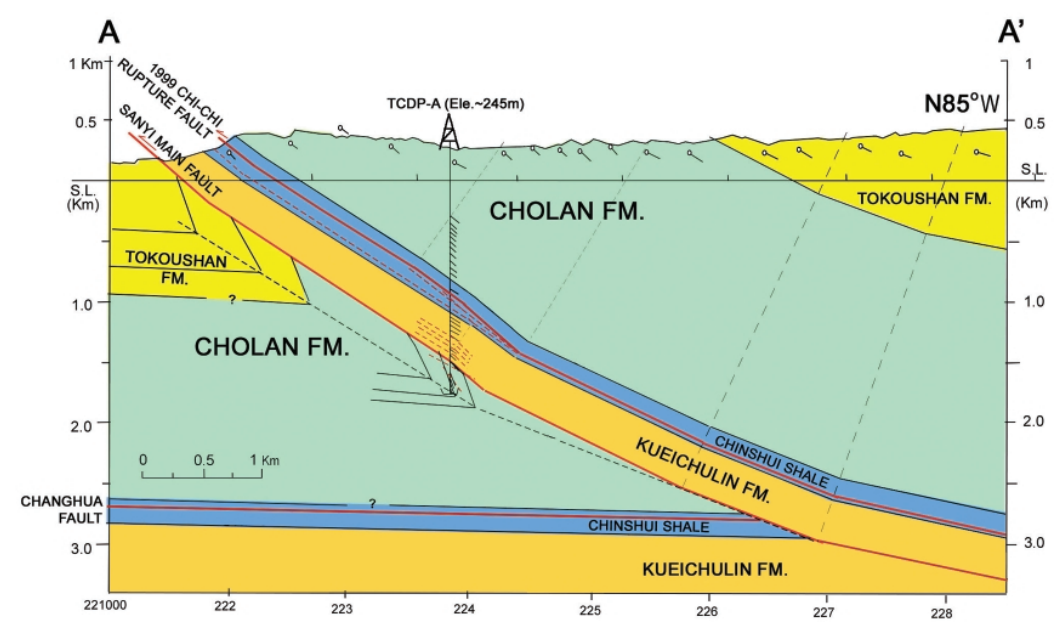

Fig. 2. E-W Cross section A A' showing the structural and stratigraphic features of Chelungpu fault. The locality of this section is shown in Fig. 1. The TCDP well-A drilled into two major thrust systems including the Chelungpu and Sanyi faults, separately.

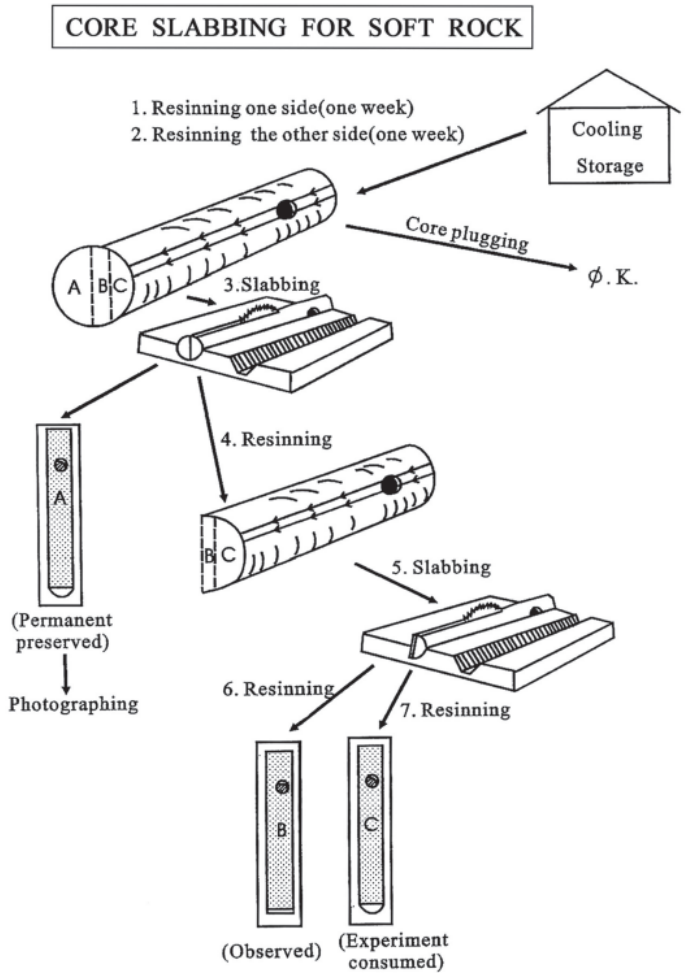

Fig. 3. Procedures for core slabbing and preservation of TCDP well-A . 
A typical example of core slabbing and a core photograph are shown in Fig. 4. Core photographs show detail of the lithology, sedimentary, and structural fabrics of the cores at different depth intervals. After pictures were taken of the entire core, close-ups were taken of the upper, middle, and lower parts of the core with overlap to allow for correlation in the digital database. The fabric in the detailed close-ups is much clear than that given by the core scanner.

\section{NANNOFOSSIL ANALYSIS}

TCDP well-A penetrates mudstone dominated strata making it difficult to discern the boundaries among the three formations. This affects the casing project and electrical logging plan during drilling. In this study, more than 150 samples were analyzed for nannofossil stratigraphy. In some cases the nature of the stratgraphy was immediately recognizable during the drilling process.

(a)

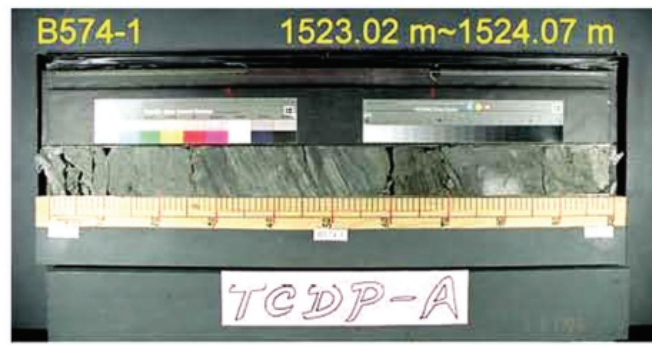

(c)

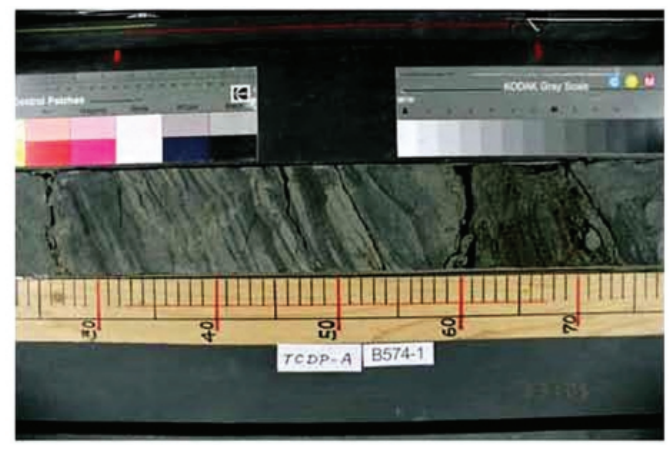

(b)

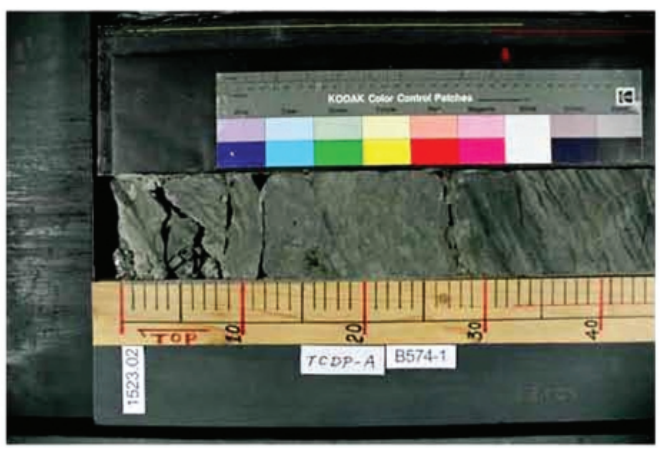

(d)

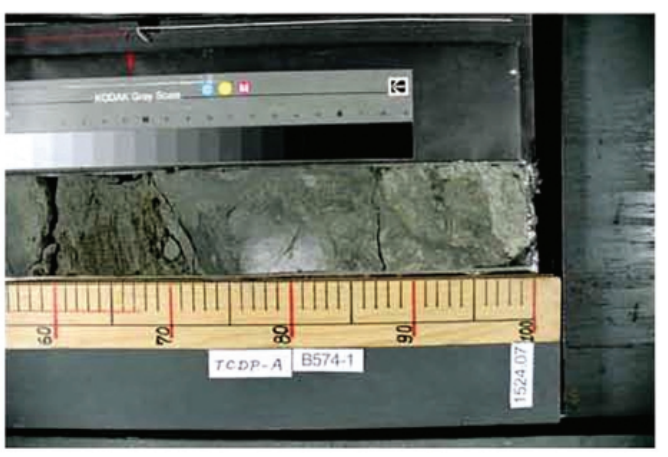

Fig. 4. Core photography: (a) photograph of the whole core; (b) amplified upper part photograph; (c) amplified middle part photograph; (d) amplified lower part photograph. The close-up photographs contain overlap between the upper, middle and lower parts for correlation purposes. 


\subsection{Methods}

Samples were taken from the marine strata whenever possible; i.e., from shale, fine grain sandstone, or mudstone. After removing any surface material, a small amount of fresh sample $(>30 \mathrm{~g})$ was bagged, numbered, and logged for depth. When confronted with geological intervals that lacked conformity, it was important that more sample points were utilized and records were taken describing the unconformity of the contact features between the overlying and underlying strata.

\subsubsection{Thin Section Preparation for Nannofossil Analysis}

In the microfossil laboratory of EDRI (CPC), Dr. Gartner's method was utilized; however, Dr. Haq's method was kept for simple and easy processing in addition to avoiding the production of poisonous gas during heating of the Caedax resin. Dr. Gartner's method is highly suitable for the concentration of samples but is limited only to calcareous nannofossils.

\subsubsection{Identification}

After finishing rock-sample preparation, identification and counting of the assembled number was accomplished using high resolution Zeiss microscopes with polarized Nicolas. The identification criteria were based on the nannofossil zonation and definition developed by Martini (1971).

\subsubsection{Fossil Photographs}

The following fossil photographs were taken using a digital camera and prepared with CoreDraw software.

\subsubsection{Fossil Statistics}

The degree of richness in the distribution of nannofossils is completed using an xy dispersive pattern from an Excel applied format. Examining the richness distribution of the nannofossils helps to determine stratigraphic boundaries within each well column.

\subsection{Illustration of Nannofossil Zones}

Based on the classification of Martini (1971) (Fig. 5), Okada and Bukry (1980), and Chi et al. (1981), there are three nannofossil zones within the Chelungpu Fault area. At present, possible fossil zones encountered in TCDP well-A are described as follows:

\section{NN12: Ceratolithus Acutus Zone}

Definition: The first appearance of Ceratolithus acutus Gartner and Bukry as the lower boundary, the first appearance of Ceartolithus rugosus Bukry \& Bramtette as the upper boundary, and all intermediate parts belong to NN12. The upper boundary determination is aided by the first appearance of Gephyrocapsa sp. 


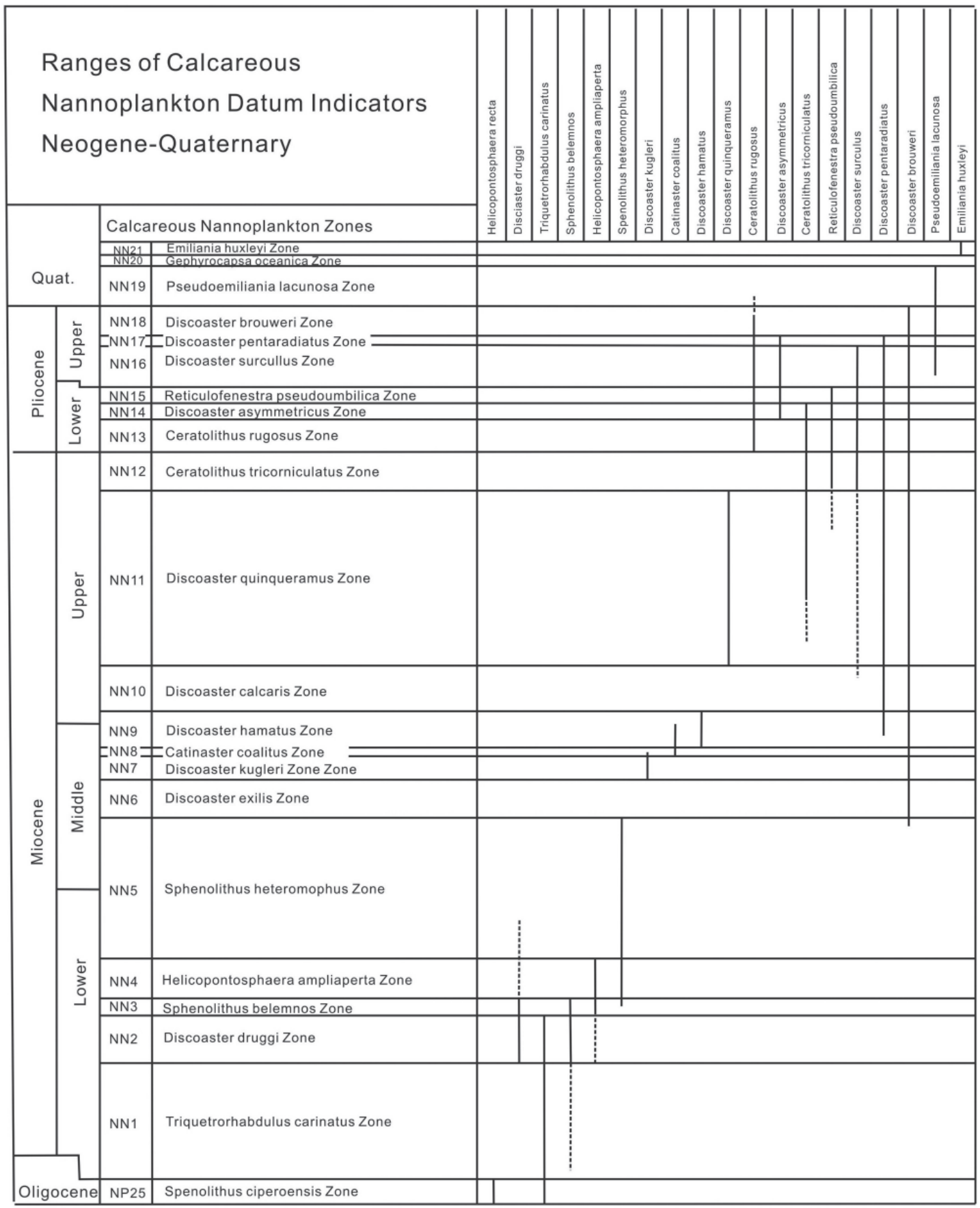

Fig. 5. Neogene and Quaternary nannofossil index in the Taichung area (after Martini 1971). 
Correlation: The NN12 zone is from early stage Pliocene and correlates the Kuantaoshan Sandstone Member to the middle part of the Shihliufen Shale Member in the Kueichulin Formation in the Chuhuangkeng profile of Miaoli area.

\section{NN13 - 15: Reticulofenstra Pseudombilica Zone}

Definition: The first appearance of Certolithus rugosus Bukry \& Bramlette as the lower boundary, the extinction surface of Reticulofenstra pseudombilica (Gartner), and all intermediate parts belong to NN13 - 15. Due to the excessive length of the existing time span of Reticulofenstra pseudombilica (Gartner) (NN7 - 15) and mixing by recycling secondary Reticulofenstra pseudombilica (Gartner) species, there are no unique characteristics specific to index fossils in the area studied. Auxiliary determination of the upper boundary is aided by the extinction surface of Sphenolithus abies Deflandre in Deflandre \& Fert. The NN13 - 15 zone is from early stage Pliocene.

Correlation: In the NN13 - 15 zone, the upper part of Shihliufen Shale and the Yutenping Sandstone Members of the Kueichulin Formation correlate to the lower part of the Chinshui Shale in the Chuhuangkeng profile of the Miaoli area.

\section{NN16 - 18: Calcidiscus Macintyrei Zone}

Definition: the extinction surface of Reticulofenstra pseudoumbilica (Gartner) as the lower boundary, the first appearance of Gephyrcapsa oceanica Kamptner as the upper boundary, and all intermediate parts belong to this zone. The extinction surface of Discoaster brouweri Bramlette \& Riedel is not used. The NN16 - 18 nannofossil zone is from late stage Pliocene. Correlation: In the NN16 - 18 zone, the upper Chinshui Shale correlates to the Cholan Formation in the Hsinchu, Miaoli, and Taichung areas.

\section{NN19: Pseudoemiliania Lacunosa Zone}

Definition: The first appearance of Gephyrocapsa oceanica Kamptner as the lower boundary, the extinction surface of Pseudoemiliania lacunosa as the upper boundary, and all intermediate parts belong to this zone. The NN19 nannofossil zone is from Pleistocene.

Correlation: In the NN19 zone, the top of Cholan Formation correlates to the Tokoushan Formation in the Hsinchu, Miaoli, and Taichung areas.

\subsection{Results of Nannofossil Analysis}

In reference to: the appearance of index fossils, the amount of nannofossils, fossil assemblages, the amount of secondary reworked fossils, lithology, and superposition of overlying and underlying strata, the 431.34 - $2003 \mathrm{~m}$ geological column of TCDP well-A can be subdivided into 12 intervals. Each of these fossil assemblages will be described in the following way (Tables 1 - 10).

Depth interval 431.34 - $869 \mathrm{~m}$ : This interval contains few fossils with no index fossils of Gephyrcapsa oceanica Kamptner found. The Gephyrcapsa oceanica Kamptner is very common in formations of the Pleistocene to Holocene; the non-existence of this fossil means that 
the strata are older than the Pleistocene. Therefore, it is necessary to refer to the underlying formation comprised of the NN15 nannofossil zone equivalent to the lower Pliocene. We deduced that the 431.34 - 869 m interval belongs to the upper Pliocene (NN16 - 18), where all the assemblages are Miocene secondary fossils such as Sphenolithus moriformis Bramlette \& Wilcoxon (1967); Sphenolithus heteromorphus Deflangre (1953); Reticulofenestra pseudoumbilica Gartner (1969c); Discoaster deflandrei Bramlette \& Sullivian (1954); Cyclicargolithus floridanus Bukry (1971a). It can be inferred from this that we didn't find index fossils of the late Pliocene and that the secondary Miocene fossils are superposed to the Pliocene.

Depth interval 882.6 - 906.1 m: Fossils are easily found in this interval. The index fossil is Sphenolithus abies Deflandre in Deflandre \& Fert (1954), the time span of which is NN7 - 15. Most assemblages are the Miocene secondary reworked fossils, such as Sphenolithus moriformis Bramlette \& Wilcoxon (1967); Sphenolithus heteromorphus Deflandre(1953); Cyclicargolithus floridanus Bukry (1971a); Discoaster deflandrei Bramlette \& Sullivian (1954). The fossils belong to the NN15 nannofossil zone which is equivalent to the early to middle stages of Pliocene.

Depth interval $914.04-1080.9 \mathrm{~m}$ : This interval contains few fossils. A small amount of the fossils are Pliocene but most of them are Miocene secondary reworked fossils.

Depth interval 1087.1 - 1099.53 m: This interval contains few fossils. The Pliocene index fossils include Sphenolithus abies Deflandre in Deflandre \& Fert (1954); Discoaster pentaradiatus Bramlette \& Riedel (1954); and Reticulofenstra pseudoumilica Gartner (1969c). The Ceratolithus sp. belong to the NN15 fossil zone and are also equivalent to the early Pliocene (Figs. 5, 7).

Depth interval 1110.25 - 1114.35 m: This interval contains few fossils. A small amount of the assemblages are Pliocene and most are Miocene secondary fossils (Figs. 5, 8).

Depth interval 1119.91 - 1151.32 m: Fossils are easily found in this interval. It consists of early Pliocene index fossils including: Sphenolithus abies Deflanfre in Deflandre \& Fert (1954); Discoaster pentaradiatus Bramlette \& Riedel (1954); and Reticulofenstra pseudoumbilica Gartner (1969c). They belong to the NN15 zone and are equivalent to the early Pliocene.

Depth interval 1157.65 - $1172.8 \mathrm{~m}$ : This interval contains few fossils. A small amount of the assemblages are Pliocene fossils; most are Miocene secondary fossils.

Depth interval 1176.9 - 1201.5 m: Fossils are easily found in this interval. The index fossils include: Sphenolithus abies Deflandre \& Fert (1954); Sphenolithus neoabies Bukry \& Bramlette (1969a); Reticulofenstra pseudoumbilica Gartner (1969c); Reticulofenstra minutula Haq \& Berggren (1978); and Discoaster pentaradiatus Bramlette \& Riedel (1954), which belong to the NN15 fossil zone and are also equivalent to the early stage of Pliocene (Figs. 5, 9).

Depth interval 1223.94 - $1266.93 \mathrm{~m}$ : This interval contains an abundance of fossils. A few Sphenolithus abies Deflanfre in Deflandre \& Fert (1954); Sphenolithus neoabies Bukry \& 
Bramlette (1969a); Reticulofenstra pseudoumbilica Gartner (1969c); Reticulofenstra minutula Haq \& Berggren (1978); Discoaster pentaradiatus Bramlette \& Riedel (1954); and Helicosphaera sellii Bukry \& Bramlette (1969b). The Gephyrocapsa sp. occupies most parts of the interval while the Reticulofenstra minutula Haq \& Berggren (1978) occupies the second part, and almost no Pseudoumbilica lacunosa Gartner (1969c) exist. This interval belongs to the NN15 fossil zone and is equivalent to early stage Pliocene.

Depth interval 1266.93 - $1297.69 \mathrm{~m}$ : This interval contains few fossils. There are a small amount of Pliocene fossils such as Sphenolithus neoabies Bukry \& Bramlette (1969a); Helicosphaera sellii Bukry \& Bramlette(1969b); and Gephyrocapsa sp., which probably belong to zone NN12 - 14 and are equivalent to early stage Pliocene.

Depth interval 1302.32 - 1710.07 m: This interval is comprised of few fossils. A small amount of Pliocene fossil assemblages exist such as Sphenolithus abies Deflanfre in Deflandre \& Fert (1954); Sphenolithus neoabies Bukry\& Bramlette (1969a); Gephyrocapsa sp.; and Pseudoumbilica lacunosa Gartner (1969c). However, in the boundary between the overlying and underlying strata there was no evidence of Miocene secondary fossils such as Sphenolithus heteromorphus Delandre (1953) and Cyclicargolithus floridanus Bukry (1971a), which are also found in the NN12 - 14 nannofossil zone and are equivalent to early to middle stage Pliocene (Figs. 5, 10).

Depth interval 1712.7 - 2003 m: This interval contains few fossils, most of which are Miocene secondary reworked fossils. The fossil assemblages are similar to those of depth interval $431.34-869 \mathrm{~m}$. The samples are softer and the cementation is less than the overlying stratum. Extending down as far as the bottom of the well, determinative index fossils such as Discoaster quinqueramus (Gartner) in the NN11 fossil zone, Sphenolithus abies Deflanfre in Deflandre \& Fert (1954) in the NN13 - 15 zone; and Gephyrocapsa oceanica Kamptner in zone NN19 were not found. Therefore, it is deduced that the nannofossil biostratigraphy between 1712.7 and $2003 \mathrm{~m}$ correlates to the upper Pliocene (Figs. 5, 11, 12).

In conclusion, the nannofossil biostratigraphy between the well top and $869 \mathrm{~m}$ corresponds to zones NN16 - 18, making this interval equivalent to the Cholan Formation. $882.6 \mathrm{~m}$ corresponds to the NN15 biozone and correlates to the Chinshui Shale. The interval $1111-1313 \mathrm{~m}$ is the contact interval with the major Chelungpu fault zone and beyond this point to a depth of $1225.7 \mathrm{~m}$ is still within in the NN15 biozone, equivalent to Chinshui Shale. The interval 1292.37 - $1710 \mathrm{~m}$ is judged to be within the NN12-NN14 biozones, correlating to the Kueichulin Formation. Below 1710 m, the well is thought to have penetrated the Sanyi Fault (Fig. 6) on account of the repeated appearance of dominate secondary reworked fossils. This interval is classified as NN16 - 18, correlating to the Cholan Formation (Figs. 5, 6).

\section{DISCUSSION}

Since Pengli orogeny began, Neogene rocks in the western basin have been deformed folding and thrusting. At the same time, reworked fossils were deposited in the younger strata.

In the sedimentary strata of the Taiwan area, the index fossils of NN12 - 14 are very rare 
Table 1. Nannofossil analysis for 431.34 - $622 \mathrm{~m}$.

\begin{tabular}{|c|c|c|c|c|c|c|c|c|c|c|c|c|c|c|c|c|c|c|c|c|c|c|c|c|c|}
\hline TCDP-A & $\frac{\vec{m}}{\vec{m}}$ & & $\frac{a}{2}$ & 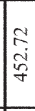 & $\begin{array}{l}\text { ป } \\
\text { ci } \\
\text { o }\end{array}$ & $\begin{array}{l}9 \\
\dot{9} \\
\end{array}$ & $\begin{array}{l}8 \\
\dot{c} \\
\dot{\infty} \\
\dot{0}\end{array}$ & $\mid \begin{array}{l}\frac{1}{3} \\
\frac{3}{9}\end{array}$ & 离 & $\left|\begin{array}{c}9 \\
0 \\
i \\
i n \\
b\end{array}\right|$ & $\begin{array}{l}\infty \\
n \\
n \\
n \\
n \\
n\end{array}$ & $\left|\begin{array}{l}0 \\
0 \\
0 \\
0 \\
0 \\
0\end{array}\right|$ & $\left|\begin{array}{l}0 \\
\\
y \\
n \\
n\end{array}\right|$ & $\left|\begin{array}{l}\frac{\infty}{\infty} \\
0 \\
\dot{0} \\
\dot{n}\end{array}\right|$ & $\begin{array}{l}8 \\
8 \\
\circ \\
n \\
n\end{array}$ & $\begin{array}{l}9 \\
0 \\
0 \\
0 \\
n\end{array} \mid$ & $\begin{array}{l}n \\
\tilde{1} \\
0 \\
\infty\end{array}$ & 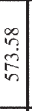 & 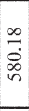 & 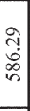 & & $\begin{array}{l}\text { 尛 } \\
0 \\
\dot{0} \\
0\end{array}$ & $\begin{array}{l}\tilde{\sigma} \\
\text { gे }\end{array}$ & & $\begin{array}{l}3 \\
\stackrel{0}{0} \\
\stackrel{0}{0}\end{array}$ \\
\hline$\triangle B U N D A N C E$ per 10 fields & 3 & ? & 2 & $\sim$ & ? & $=$ & - & 0 & $\because$ & $\circ$ & $\stackrel{3}{0}$ & - & 0 & -5 & $\overline{0}$ & 0 & 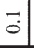 & $\overline{0}$ & 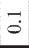 & 0 & 0 & - & $\overrightarrow{0}$ & $\overrightarrow{0}$ & $\overrightarrow{0}$ \\
\hline \multicolumn{26}{|l|}{ Amaurolithus amplificus } \\
\hline A. delicatus & & & & & & & & & & & & & & & & & & & & & & & & & \\
\hline A. primus & & & & & & & & & & & & & & & & & & & & & & & & & \\
\hline A. tricorniculatus & & & & & & & & & & & & & & & & & & & & & & & & & \\
\hline Coccolithus pelagicus & & & & $\mathrm{R}$ & R & & & & $\ddot{P}$ & & & & & P & & & & & $\mathrm{P}$ & & & $\dddot{P}$ & $\mathrm{P}$ & & $P$ \\
\hline Catinaster calyculus & & & & & & & & & & & & & & & & & & & & & & & & & \\
\hline C. coalitus & & & & & & & & & & & & & & & & & & & & & & & & & \\
\hline Ceratolithus acutus & & & & & & & & & & & & & & & & & & & & & & & & & \\
\hline C. armatus & & & & & & & & & & & & & & & & & & & & & & & & & \\
\hline C. cristatus & & & & & & & & & & & & & & & & & & & & & & & & & \\
\hline C. rugosus & & & & & & & & & & & & & & & & & & & & & & & & & \\
\hline Coronocyclus nitescens & & & & & & & & & & & & & & & & & & & & & & & & & \\
\hline Cyclicargolithus floridanus & $\mathrm{R}$ & & & $\mathrm{R}$ & R & & 2 & & & & R & & & $P$ & D & & & & $P$ & & & $P$ & & & \\
\hline C. abisectus & & & & & & & & & & & & & & & & & & & & & & & & & \\
\hline Calcidiscus leptoporus & & & & & & & & & & & & & & & & & & & & & & & & & \\
\hline C. macintyrei & & & & & & & & & & & & & & & & & & & & & & & & & \\
\hline Dictyococcites bisectus & & & & & & & & & & & & & & & & & & & & & & & & & \\
\hline D. productus & & & & & & & & & & & & & & & & & & & & & & & & & \\
\hline Discoaster adamanteus & & & & & & & & & & & & & & & & & & & & & & & & & \\
\hline D. asymmetricus & & & & & & & & & & & & & & & & & & & & & & & & & \\
\hline D. bellus & & & & & & & & & & & & & & & & & & & & & & & & & \\
\hline D. bollii & & & & & & & & & & & & & & & & & & & & & & & & & \\
\hline D. brouweri & & & & & & & & & & & & & & & & & & & & & & & & & \\
\hline D. calcaris & & & & & & & & & & & & & & & & & & & & & & & & & \\
\hline D. deflandrei & & & & & & & & & & & & & & & & & & & & & & & & & \\
\hline D. druggi & 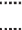 & & & & & & & & & & & & & & & & & & & & & & & & \\
\hline D. exilis & & & & & & & & & & & & & & & & & & & & & & & & & \\
\hline D. hamatus & & & & & & & & & & & & & & & & & & & & & & & & & \\
\hline D. neohamatus & & & & & & & & & & & & & & & & & & & & & & & & & \\
\hline D. pentaradiatus & $\cdots$ & & & & & & & & & & & & & & & & & & & & & & & & \\
\hline D. quinqueramus & & & & & & & & & & & & & & . & & & & & & & & & & & \\
\hline D. surculus & ....... & & & & ........ & & & & & & & & & & & & & & & & & & & & \\
\hline D. tamalis & & & & & & & & & & & & & & & & & & & & & & & & & \\
\hline D. variabilis & & & & & $\mathrm{P}$ & & & & & & & & & & & & & & & & & & & & \\
\hline Emiliania huxleyi & & & & & & & & & & & & & & & & & & & & & & & & & \\
\hline Ericsonia fenestrata & & & & & & & & & & & & & & & & & & & & & & & & & \\
\hline Gephyrocapsa spp. & (es & & & Pe' & wis & & $\vdots$ & & & & 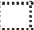 & & & 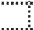 & & & & 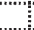 & & & & & 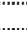 & & (') \\
\hline G. oceanica & & & & & & & & & & & & & & & & & & & & & & & & & \\
\hline Helicosphoera ampliaperta & & & & .... & & & & & & & $\ldots$ & & & & & & & & & & & & & & \\
\hline H. carteri & & & & & & & & & & & 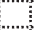 & & & & & & & & & & & & & & \\
\hline H. intermedia & & & & $\cdots$ & 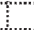 & & & & & & ...' & & & & & & & & & & & & & & \\
\hline H. obliqua & & & & & & & & & & & & & & & & & & & & & & & & & \\
\hline H. sellii & & & & & ".' & & se & & & & R & & & & & & & & & & & & $\mathrm{P}$ & & \\
\hline Oolithotus fragilis & & & & & & & & & & & & & & & & & & & & & & & & & \\
\hline Pseudoemiliania lacunosa & & & & & & & & & & & & & & & & & & & & & & & & & \\
\hline Reticulofenestra spp. & R & $\mathrm{R}$ & $R$ & R & $R$ & $\mathrm{VR}$ & $R$ & & P & & $\mathrm{R}$ & & & & & & & & & & & & 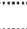 & & .... \\
\hline R. minutula & & $\mathrm{R}$ & $R$ & $R$ & $\mathrm{R}$ & & $R$ & & & & $R$ & & & & & & & & & & & & $\mathrm{P}$ & & \\
\hline R. pseudoumbilica & & & $R$ & R & $\mathrm{R}$ & & $R$ & & & & & & & & & & & $\mathrm{P}$ & & & & & & & $\mathrm{P}$ \\
\hline Rhabdosphaera clavigera & & & & & & & & & & & & & & & & & & & & & & & & & \\
\hline Sphenolithus abies & & & & & & & & & & & & & & & & & & & & & & & & & \\
\hline S. belemnos & & & & & & & & & & & & & & & & & & & & & & & $\mathrm{P}$ & & \\
\hline S. ciperoensis & & & & & & & & & & & & & & & & & & & & & & & & & \\
\hline S. conicus & & & & & & & & & & & & & & & & & & & & & & & & & \\
\hline S. dissimilis & & & & & & & & & & & & & & & & & & & & & & & & & \\
\hline S. distentus & & & & & & & & & & & & & & 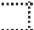 & & & & & & & & & & & 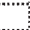 \\
\hline S. heteromorphus & R & & & $R$ & $R$ & & $R$ & & & & $R$ & & & & & & & & & & & & & $\cdots$ & \\
\hline S. moriformis & & & & & & & & & & & & & & $\mathrm{P}$ & & & & & & & & & & & $P$ \\
\hline S. neoabies & & & & & & & & & & & & & & & & & & $\mathrm{P}$ & $\mathrm{P}$ & & & & & $\mathrm{P}$ & \\
\hline Syracosphaera histrica & & & & & & & & & & & & & & & & & & & & & & & & & \\
\hline Umbilicosphaera sibogae & & & & & & & & & & & & & & & & & & e & & & & & & 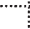 & \\
\hline
\end{tabular}


Table 2. Nannofossil analysis for $625-772.38 \mathrm{~m}$.

\begin{tabular}{|c|c|c|c|c|c|c|c|c|c|c|c|c|c|c|c|c|c|c|c|c|c|c|c|c|c|}
\hline TCDP-A & สู่ & â & 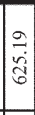 & $\frac{6}{3}$ & $\frac{2}{2}$ & 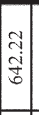 & $\begin{array}{l}\vec{\infty} \\
0 \\
\tilde{f} \\
0\end{array}$ & 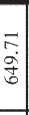 & 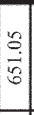 & $\mid \begin{array}{l}2 \\
2 \\
\dot{1} \\
6\end{array}$ & $\begin{array}{l}\infty \\
\infty \\
0 \\
6\end{array} \mid$ & $\begin{array}{l}n \\
\vdots \\
\hat{0} \\
0\end{array}$ & $\left|\begin{array}{l}2 \\
\vdots \\
0 \\
0\end{array}\right|$ & $\left|\begin{array}{l}2 \\
0 \\
0 \\
0 \\
0\end{array}\right|$ & $\begin{array}{l}0 \\
+ \\
+ \\
6\end{array}$ & $\begin{array}{l}0 \\
\dot{0} \\
0\end{array}$ & $\begin{array}{l}0 \\
\dot{0} \\
0 \\
0\end{array}$ & : & $\begin{array}{l}0 \\
m \\
\vdots \\
\vdots\end{array}$ & $\begin{array}{l}\text { गे } \\
\text { ถે }\end{array}$ & $\begin{array}{l}\mathrm{n} \\
\mathrm{n}\end{array}$ & & 点 & & \\
\hline ABUNDANCE per 10 fields & - & 4 & $\overrightarrow{0}$ & 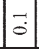 & 0 & 0 & o & 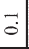 & 0 & $\overrightarrow{0}$ & ֻุ| & $\overrightarrow{0}$ & o & 0 & 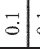 & $\overrightarrow{0}$ & รี) & $\circ$ & $\overline{0}$ & $\overrightarrow{0}$ & $\circ$ & o & o & o & \\
\hline Amaurolithus amplificus & & & & & & & & & & & & & & & & & & & & & & & & & \\
\hline A. delicatus & & & & & & & & & & & & & & & & & & & & & & & & & \\
\hline A. primus & & & & & & & & & & & & & & & & & & & & & & & & & \\
\hline A. tricorniculatus & & & & & & & & & & & & & & & & & & & & & & & & & \\
\hline Coccolithus pelagicus & & & & $p$ & & & & & & & & $P$ & & & & & & & $\mathrm{P}$ & & & & & & \\
\hline Catinaster calyculus & & & & & & & & & & & & & & & & & & & & & & & & & \\
\hline C. coalitus & & & & & & & & & & & & & & & & & & & & & & & & & \\
\hline Ceratolithus acutus & & & & & & & & & & & & & & & & & & & & & & & & & \\
\hline C. armatus & & & & & & & & & & & & & & & & & & & & & & & & & \\
\hline C. cristatus & & & & & & & & & & & & & & & & & & & & & & & & & \\
\hline C. rugosus & & & & & & & & & & & & & & & & & & & & & & & & & \\
\hline Coronocyclus nitescens & & & & & & & & & & & & & & & & & & & & & & & & & \\
\hline Cyclicargolithus floridanus & $\mathrm{R}$ & $\mathrm{R}$ & & $P$ & & & & & & & & & & & & & & & & & & & & & \\
\hline C. abisectus & $P$ & $\mathrm{P}$ & & & & & & & & & & & & & & & & & & & & & & & \\
\hline Calcidiscus leptoporus & & $P$ & & & & & & & & & & & & & & & & & & & & & & & \\
\hline C. macintyrei & & $\mathrm{P}$ & & & & & & & & & & & & & & & & & & & & & & & \\
\hline Dictyococcites bisectus & & & & & & & & & & & & & & & & & & & ... & P & & & & & \\
\hline D. productus & & & & & & & & & & & & & & & & & & & & & & & & & \\
\hline Discoaster adamanteus & & $P$ & & & & & & & & & & & & & & & & & & & & & & & \\
\hline D. asymmetricus & & $\ldots$ & & & & & & & & & & & & & & & & & & & & & & & \\
\hline D. bellus & & 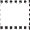 & & & & & & & & & & & & & & & & & 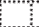 & & & & & & \\
\hline D. bollii & & $\ldots$ & & & & & & & & & & & & & & & & & & & & & & & \\
\hline D. brouweri & & & & & & & & & & & & & & & & & & & & & & & & & \\
\hline D. calcaris & & & & & & & & & & & & & & & & & & & & & & & & & \\
\hline D. deflandrei & & $\mathrm{R}$ & & & & & & & & & & & & & & & & & & & & & & & \\
\hline D. druggi & P & $\mathrm{P}$ & & & & & & & & & & & & & & & $\ldots$ & $\ldots$ & & & & & & & \\
\hline D. exilis & & & & & & & & & & & & & & & & & & & & & & & & & \\
\hline D. hamatus & (i) & 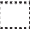 & & & & & & & & & & & & & & & & & & & & & & & \\
\hline D. neohamatus & & & & & & & & & & & & & & & & & & & & & & & & & \\
\hline D. pentaradiatus & ....... & & & & & & & & & & & & & & & & & & & & & & & & \\
\hline D. quinqueramus & & ... & & & & & & & & & & & & & & & & & & & & & & & \\
\hline D. surculus & & & & & & & & & & & & & & & & & & & & & & & & & \\
\hline D. tamalis & & & & & & & & & & & & & & & & & & & & & & & & & \\
\hline D. variabilis & & & & & & & & & & & & & & & & & & & & & & & & & \\
\hline Emiliania huxleyi & & & & & & & & & & & & & & & & & & & & & & & & & \\
\hline Ericsonia fenestrata & & & & & & & & & & & & & & & & & & & & & & & & & \\
\hline Gephyrocapsa spp. & & $\mathrm{P}$ & & & & & & & & & & & & & & R & & & $p$ & & & & & & \\
\hline G. oceanica & & & & & & & & & & & & & & & & & & & & & & & & & \\
\hline Helicosphoera ampliaperta & & & & & & & & & & & & & & & & & & & & & & & & & \\
\hline H. carteri & . & 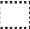 & & & & & & & & & & & & & & & 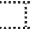 & & & & & & & & \\
\hline H. intermedia & (3) & & & & & & & & & & & & & & & & & & & & & & & & \\
\hline H. obliqua & & & & 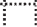 & & & & & & & & & & & & & (a) & & & & & & & & \\
\hline H. sellii & & $\mathrm{P}$ & & & & & & & & & & $\mathrm{P}$ & & & & & & & & & & & & & \\
\hline Oolithotus fragilis & & & & & & & & & & & & & & & & & & & & & & & & & \\
\hline Pseudoemiliania lacunosa & & & & & & & & & & & & & & & & & & & & & & & & & \\
\hline Reticulofenestra spp. & R & $\mathrm{R}$ & & & & & & & & & & & & & & & R & & & & & & & & ?. \\
\hline R. minutula & & & $\mathrm{P}$ & $R$ & & & & $\mathrm{P}$ & & $P$ & $\mathrm{VR}$ & $P$ & & & & & & & & & & & & & \\
\hline R. pseudoumbilica & & $\mathrm{R}$ & $\mathrm{P}$ & $R$ & & & & & & $\mathrm{p}$ & & $P$ & & & $R$ & & P & & & & & & & & \\
\hline Rhabdosphaera clavigera & & & & & & & & & & & & & & & & & & & & & & & & & \\
\hline Sphenolithus abies & & & & & & & & & & & & & & & & & & & & & & & & & \\
\hline S. belemnos & (.) & & $\mathrm{P}$ & & & & & & & & & & & & & & & & & & & & & & \\
\hline S. ciperoensis & & & & & & & & & & & & & & & & & & & & & & & & & \\
\hline S. conicus & & & & & & & & & & & & & & & & & P̈ & & & & & & & & \\
\hline S. dissimilis & & & & & & & & & & & & & & & & & & & & & & & & & \\
\hline S. distentus & & & & & & & & & & & & & & & & & & & & & & & & & \\
\hline S. heteromorphus & R & $R$ & & & & & & & & & $\mathrm{VR}$ & & & & & & & & & & & & & & \\
\hline S. moriformis & P & $R$ & & $\mathrm{P}$ & & & & & & $P$ & & & & & & & & & $P$ & & & & & & \\
\hline S. neoabies & & $\mathrm{P}$ & & & & & & & & & & & & & & & & & & & & & & & \\
\hline era histrica & & & & & & & & & & & & & & & & & & & & & & & & & \\
\hline Umbilicosphaera sibogae & & & & & & & & & & & & & & & & & & & & & & & & & \\
\hline
\end{tabular}


Table 3. Nannofossil analysis for $780.66-1032.6 \mathrm{~m}$.

\begin{tabular}{|c|c|c|c|c|c|c|c|c|c|c|c|c|c|c|c|c|c|c|c|c|c|c|c|c|c|}
\hline TCDP-A & 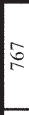 & 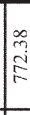 & 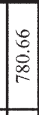 & $\mid \begin{array}{l}0 \\
\vdots \\
\vdots \\
2 \\
2\end{array}$ & $\mid \begin{array}{l}n \\
0 \\
\dot{0} \\
0\end{array}$ & 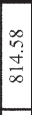 & $\begin{array}{l}m \\
\stackrel{2}{*}\end{array}$ & $\begin{array}{l}\infty \\
\dot{0} \\
\dot{\infty}\end{array}$ & $\left|\begin{array}{c}0 \\
0 \\
0 \\
\vdots \\
\infty\end{array}\right|$ & $\begin{array}{l}\infty \\
0 \\
\vdots \\
\vdots \\
\infty \\
\infty\end{array}$ & 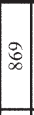 & $\begin{array}{l}0 \\
\tilde{i} \\
\infty \\
\infty\end{array}$ & 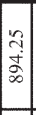 & $\left|\begin{array}{l}2 \\
\vdots \\
2 \\
\infty \\
\infty\end{array}\right|$ & 㝘 & $\begin{array}{l}\tilde{0} \\
\dot{t} \\
\sigma\end{array} \mid$ & $\begin{array}{l}- \\
0 \\
2\end{array}$ & $\begin{array}{l}0 \\
\dot{z} \\
\dot{f}\end{array}$ & $\begin{array}{l}0 \\
\text { ț. } \\
\text { 2. }\end{array}$ & $\begin{array}{l}\vec{\vdots} \\
\text { ळे }\end{array}$ & $\begin{array}{l}y \\
\infty \\
2\end{array}$ & 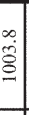 & & & \\
\hline ABUNDANCE per 10 fields & $\infty$ & $\overrightarrow{0}$ & 0 & $\overline{0}$ & $\circ$ & $\overrightarrow{0}$ & 0 & $\approx$ & 0 & $\circ$ & $\ddot{0}$ & $\overrightarrow{4}$ & 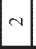 & $\simeq$ & $\sim$ & 0 & ชิ & $\circ$ & $\circ$ & ชู. & $m$ & 0 & 0 & & 0 \\
\hline Amaurolithus amplificus & & & & & & & & & & & & & & & & & & & & & & & & & \\
\hline A. delicatus & & & & & & & & & & & & & & & & & & & & & & & & & \\
\hline A. primus & & & & & & & & & & & & & & & & & & & & & & & & & \\
\hline A. tricorniculatus & & & & & & & & & & & & & & & & & & & & & & & & & \\
\hline Coccolithus pelagicus & & & & & & $\mathrm{P}$ & & & & & & & & & & & $P$ & & & & & & & & \\
\hline Catinaster calyculus & & & & & & & & & & & & & & & & & & & & & & & & & \\
\hline C. coalitus & & & & & & & & & & & & & & & & & & & & & & & & & \\
\hline Ceratolithus acutus & & & & & & & & & & & & & & & & & & & & & & & & & \\
\hline C. armatus & & & & & & & & & & & & & & & & & & & & & & & & & \\
\hline C. cristatus & & & & & & & & & & & & & & & & & & & & & & & & & \\
\hline C. rugosus & & & & & & & & & & & & & & & & & & & & & & & & & \\
\hline Coronocyclus nitescens & & & & & & & & & & & & & & & & & & & & & & & & & \\
\hline Cyclicargolithus floridanus & & $P$ & & & & & & & & & & $p$ & $p$ & & & & & & & & & & & & \\
\hline C. abisectus & & & & & & & & & & & & & & & & & & & & & & & & & \\
\hline Calcidiscus leptoporus & & & & & & & & & & & & & & & & & & & & & & & & & \\
\hline C. macintyrei & & & & & & & & & & & & & & & & & & & & & $\mathrm{P}$ & & & & \\
\hline Dictyococcites bisectus & & & & & & & & & & & & & & & & & & & & & & & & & \\
\hline D. productus & & & & & & & & & & & & & & & & & & & & & & & & & \\
\hline Discoaster adamanteus & & & & & & & & & & & & - & & & & & & & & & & & & & \\
\hline D. asymmetricus & & & & & & & & & & & & 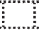 & & & & & & & 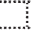 & 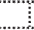 & & & & & \\
\hline D. bellus & & & & & & & & & & & & & & & & & & & & & & & & & \\
\hline D. bollii & & & & & & & & & & & & & & & & & & & & 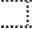 & & & & & \\
\hline D. brouweri & & & & & & & & & & & & & & & & & & & & & .... & & & & \\
\hline D. calcaris & & & & & & & & & & & & & & & & & & & & & & & & & \\
\hline D. deflandrei & & & & & & & & & & & & & & & & & & & & ... & P.. & & & & \\
\hline D. druggi & & & & & & & & & & & & & & & & & & & & & & & & & \\
\hline D. exilis & & & & & & & & & & & & & & & & & & & & & & & & & \\
\hline D. hamatus & & & & & & & & & & & & & & & & & & & & & & & & & \\
\hline D. neohamatus & & & & & & & & & & & & & & & & & & & & 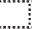 & & & & & \\
\hline D. pentaradiatus & & & & & & & & & & & & & & & & & & & & & & & & & \\
\hline D. quinqueramus & & & & & & & & & & & & . & & & & & & & & & & & & & \\
\hline D. surculus & & & & & & & & & & & & & & & & & & & & & .... & & & & \\
\hline D. tamalis & & & & & & & & & & & & & & & & & & & & 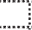 & 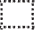 & & & & \\
\hline D. variabilis & & & & & & & & & & & & & & & & & & & & & & & & & \\
\hline Emiliania huxleyi & & & & & & & & & & & & & & & & & & & & & $\ldots$ & & & & \\
\hline Ericsonia fenestrata & & & & & & & & & & & & & & & & & & & & ( & - & & & & \\
\hline Gephyrocapsa spp. & 㙂 & $\mathrm{P}$ & & $\mathrm{P}$ & & & & & & & & $R$ & $R$ & $R$ & $R$ & & & & & & $\mathrm{P}$ & & & & \\
\hline G. oceanica & & & & & & & & & & & & & & & & & & & & & & & & & \\
\hline Helicosphoera ampliaperta & & & & & & & & & & & & & & & & & & & & & & & & & \\
\hline H. carteri & & & & & & & & & & & & & & & & & & & & $\ldots$ & $\vdots$ & & & & \\
\hline H. intermedia & & & & & & & & & & & & & & & & & & & & & & & & & \\
\hline H. obliqua & & & & & & & & & & & & & & & & & & & & & & & & & \\
\hline H. sellii & & & & & & & & & & & & & & & & & & & & $P$ & & & & & \\
\hline Oolithotus fragilis & & & & & & & & & & & & & & & & & & & & & & & & & \\
\hline Pseudoemiliania lacunosa & & & & & & & & & & & & & & & & & & & & & $P$ & & & & \\
\hline Reticulofenestra spp. & $F$ & & & & & & & & & & $\mathrm{P}$ & $\mathrm{F}$ & $\mathrm{R}$ & $R$ & & & $R$ & & & & $\mathrm{R}$ & & & & \\
\hline R. minutula & 垭 & & & P & & $R$ & & $F$ & & & $P$ & $F$ & $R$ & R & $R$ & & $R$ & & & $R$ & $R$ & & & & \\
\hline R. pseudoumbilica & & & & & & & & & & & & $R$ & & & $R$ & & & & & & & & & & \\
\hline Rhabdosphaera clavigera & & & & & & & & & & & & & & & & & & & & & & & & & \\
\hline Sphenolithus abies & & & & & & & & & & & & & & $R$ & & & P... & & & & & & & & \\
\hline S. belemnos & & & & & & & & & & & & & & & & & & & & & & & & & \\
\hline S. ciperoensis & & & & & & & & & & & & & & & & & & & & & & & & & \\
\hline S. conic & & & & & & & & & & & & & & & & & & & & & & & & & \\
\hline S. dissimilis & & & & & & & & & & & & & & & & & & & & & & & & & \\
\hline S. distentus & & & & & & & & & & & & & & & & & & & & & $P$ & & & & \\
\hline S. heteromorphus & & & & & & & & & & & & & & & & & & & & & & & & & \\
\hline S. moriformis & & & & & & & & & & & & & & & & & & P & & & & & & & \\
\hline S. neoabies & & & & & & & & & & & & & & & & & & & & & & & & & \\
\hline histrica & & & & & & & & & & & & & & & & & & & & & & & & & \\
\hline aera sibogae & & & & & & & & & & & & 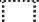 & 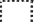 & & & & & ( & & 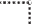 & & & & & . \\
\hline
\end{tabular}


Table 4. Nannofossil analysis for $1040.1-1139.59 \mathrm{~m}$.

\begin{tabular}{|c|c|c|c|c|c|c|c|c|c|c|c|c|c|c|c|c|c|c|c|c|c|c|c|c|c|}
\hline TCDP-A & 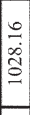 & 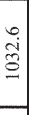 & 章 & 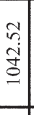 & 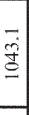 & $\overrightarrow{\underline{\sigma}}$ & है & $\stackrel{\overline{8}}{8}$ & $\begin{array}{l}\overrightarrow{\mathrm{C}} \\
\stackrel{\mathscr{O}}{\varrho}\end{array}$ & $\begin{array}{l}n \\
n \\
0 \\
0 \\
0\end{array}$ & $\begin{array}{l}\overrightarrow{0} \\
\stackrel{0}{0} \\
.\end{array}$ & $\begin{array}{l}\hat{\vdots} \\
\stackrel{8}{=} \\
=\end{array}$ & $\mid \begin{array}{l}-1 \\
\vec{\infty} \\
0 \\
=\end{array}$ & 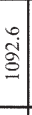 & $\overrightarrow{+}$ & $\begin{array}{l}\overrightarrow{0} \\
\dot{0} \\
-\end{array}$ & $\begin{array}{l}3 \\
2 \\
2 \\
0 \\
=\end{array}$ & & 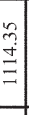 & $\begin{array}{l}\vec{\sigma} \\
\stackrel{\Xi}{\Xi} \\
\end{array}$ & 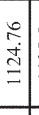 & 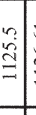 & 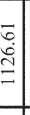 & & \\
\hline ABUNDANCE per 10 fields & in & 2 & 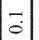 & $\overrightarrow{0}$ & o & 8 & o & o & 0 & 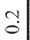 & $\overline{0}$ & $n$ & $q$ & $=$ & - & 志 & $\overline{\mathrm{N}}$ & 3 & $\overline{0}$ & $n$ & - & $\hat{\imath}$ & - & $\approx$ & $\vec{v}$ \\
\hline Amaurolithus amplificus & & & & & & & & & & & & & & & & & & & & & & & & & \\
\hline A. delicatus & & & & & & & & & & & & & & & & & & P & & & & & & & \\
\hline A. primus & & & & & & & & & & & & & & & & & & & & & & & & & \\
\hline A. tricorniculatus & & & & & & & & & & & & & & & & & & & & & & & & & \\
\hline Coccolithus pelagicus & & & & & & & & & & & & & & & & & & & & & & & & & \\
\hline Catinaster calyculus & & & & & & & & & & & & & & & & & & & & & & & & & \\
\hline C. coalitus & & & & & & & & & & & & & & & & & & & & & & & & & \\
\hline Ceratolithus acutus & & & & & & & & & & & & & & & & & & & & & & & & & $\ldots$. \\
\hline C. armatus & & & & & & & & & & & & & & $\ldots$ & & & & & & & & & & & \\
\hline C. cristatus & & & & & & & & & & & & & & & & & & & & & & & & & \\
\hline C. rugosus & & & & & & & & & & & & & & P & & & & $\mathrm{P}$ & & & & & & & \\
\hline Coronocyclus nitescens & & & & & & & & & & & & & & & & & & & & $\ldots$ & & & & & ... \\
\hline Cyclicargolithus floridanus & & P.... & & & & & & & & & & R & R.... & & & & & & & .... & & $\ldots$ & .... & & \\
\hline C. abisectus & & & & & & & & & & & & & & & & & & & & ( & & & & & \\
\hline Calcidiscus leptoporus & & & & & & & & & & R. & & & & $\ldots$ & & & & & & $\ldots$ & & & & & \\
\hline C. macintyrei & & & & & & & & & & & & & & & & & & & & $\ldots$ & & & & & \\
\hline Dictyococcites bisectus & & & & & & & & & & & & & & & & & & & & & & & & & \\
\hline D. productus & & & & & & & & & & & & & & & & & & & & (- & & & & & \\
\hline Discoaster adamanteus & & & & & & & & & & & & & & $\ldots$ & & & & & & $\cdots$ & & & & & ...... \\
\hline D. asymmetricus & & & & & & & & & & & & & & $\ldots$ & & & $\ldots$ & & & .... & & .... & $\ldots$ & & $\ldots$ \\
\hline D. bellus & & & & & & & & & & & & ... & & $\ldots$ & & & $\ldots$ & & & $\ldots$ & & $\ldots$ & $\ldots$ & & \\
\hline D. bollii & & & & & & & & & & & & & & & & & & & & & & & & & \\
\hline D. brouweri & & & & & & & & & & & & & & & & & & & & . & & & & & \\
\hline D. calcaris & & & & & & & & & & & 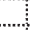 & 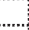 & & & & & $\ldots$ & & & .... & & & .... & & $\ldots$ \\
\hline D. deflandrei & .... & & & & & & & & & $\ldots$ & 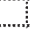 & .... & $\ldots$ & $\ldots$ & $\ldots$ & & $\ldots$ & & & .... & & & $\ldots$ & & \\
\hline D. druggi & & & & & & & & & & & & & & & & & & & & & & & & & \\
\hline D. exilis & & & & & & & & & & & & & & & & & & & & ... & & & & & \\
\hline D. hamatus & ... & & & & & & & & & & & & & 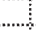 & & & $\ldots$ & & & .... & & & ... & & \\
\hline D. neohamatus & .... & & & & & & & & & & & & & & & & 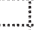 & & & & & & $\ldots$ & & \\
\hline D. pentaradiatus & ...... & & & & & & & & & & & & & $\mathrm{R}$ & R.... & & & & & $P$ & & & & & \\
\hline D. quinqueramus & ?....... & & & & & & & & & & & & $\ldots . .$. & 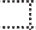 & & & & & & .... & & & $\ldots$ & & \\
\hline D. surculus & ...... & & & & & & & & & & & & & .... & & & & & & ... & & & & & \\
\hline D. tamalis & ...... & & & & & & & & & & & & $\ldots$ & ..... & & & & & & $\ldots$ & & & $\ldots$ & & \\
\hline D. variabilis & & & & & & & & & & & & & & & & & & & & & & & & & \\
\hline Emiliania huxleyi & & & & & & & & & & & & & $\ldots$ & & & & & & & & & & & & \\
\hline Ericsonia fenestrata & & & & & & & & & & & & & & & & & & & & & & & ... & & \\
\hline Gephyrocapsa spp. & $R$ & & & & & $R$ & & & & & & & $\mathrm{~F}$ & F & $R$ & c & $\mathrm{FC}$ & & & $\ldots$ & & $\ldots$ & $\ldots$ & & \\
\hline G. oceanica & & & & & & & & & & & & & & & & & & & & & & & & & \\
\hline Helicosphoera ampliaperta & & & & & & & & & & & & & & & & .... & & & & & & & & & \\
\hline H. carteri & .... & & & & & & & & & & & & R & $R$ & & ...... & & & & $\cdots$ & & ... & $\ldots$ & & .... \\
\hline H. intermedia & & & & & & & & & & $\cdots$ & & ..... & $\ldots$ & 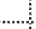 & ........ & ..... & ..... & $\cdots$ & & ....... & & $\ldots$ & ...... & $\ldots$ & 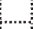 \\
\hline H. obliqua & & & & & & & & & & & & & 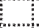 & & & 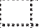 & & & & ....... & & & ...... & & \\
\hline H. sellii & & & & & & & & & & & & & & & & & & & & & & & & & \\
\hline Oolithotus fragilis & & & & & & & & & & & & & $\ldots$ & & & $\ldots$ & & & & 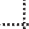 & & $\ldots$ & & & 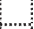 \\
\hline Pseudoemiliania lacunosa & & & & & & & & & & & & & & & & & & & & & & & & & \\
\hline Reticulofenestra spp. & $R$ & $\mathrm{R}$ & & & & & & & & $R$ & P & $R$ & $\mathrm{~F}$ & $\mathrm{~F}$ & $\mathrm{R}$ & FC: & $\mathrm{F}$ & & & $R$ & $R$ & $R$ & $\mathrm{R}$ & 2 & $\mathrm{~F}$ \\
\hline R. minutula & $R$ & $\mathrm{R}$ & & $\mathrm{R}$ & & & & & 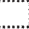 & & & $R$ & R & $R$ & $\mathrm{R}$ & $R$ & & & & R & & Re & & 3 & $F$ \\
\hline R.pseudoumbilica & & $R$ & & & & & & & & & & & $R$ & & & & & & & & & & 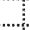 & & ........ \\
\hline Rhabdosphaera clavigera & & & & & & & & & & & & & & & & & & & & & & & & & \\
\hline Sphenolithus abies & & $R$ & & & & $P$ & & & 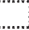 & & & $R$ & & R & $R$ & $P$ & & & & 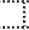 & & 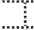 & & 2 & \\
\hline S. belemnos & & & & & & & & & & & & & $\ldots$ & ... & & .... & & & & $\ldots$ & & ... & & $\ldots$ & \\
\hline S. ciperoensis & & & & & & & & & & & & & & & & & & & $\ldots$ & 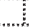 & & se & & & .... \\
\hline S. conicus & & & & & & & & & & & & & & & & & & & & & & & & & \\
\hline S. dissimilis & & & & & & & & & & & & & & & & & & & & & & & & & \\
\hline S. distentus & & & & & & & & & & & & & & & & & & & & & & & & & \\
\hline S. heteromorphus & $p$ & & & & & & & & & $\mathrm{P}$ & & & $\mathrm{P}$ & $R$ & & & & & $P$ & (a) & & ( & & ? & \\
\hline S. moriformis & & & & & & & & & & & & & $R$ & & & & & & & & & & & & \\
\hline S. neoabies & $\mathrm{P}$ & $\mathrm{R}$ & & & & & & & & & & & & R & & & & & & $\mathrm{R}$ & & R & & 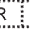 & \\
\hline Syracosphaera histrica & & & & & & & & & & & & & & & & & & & & & & & & & \\
\hline Umbilicosphaera sibogae & & & & & & & & & & & & & & & & & & & & & & & & & \\
\hline
\end{tabular}


Table 5. Nannofossil analysis for $1142.3-1232.5 \mathrm{~m}$.

\begin{tabular}{|c|c|c|c|c|c|c|c|c|c|c|c|c|c|c|c|c|c|c|c|c|c|c|c|c|c|}
\hline TCDP-A & 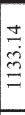 & 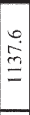 & के & 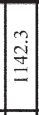 & $\begin{array}{c}a \\
\stackrel{\rho}{\tilde{z}} \\
=\end{array}$ & $\mid \begin{array}{c}2 \\
\frac{2}{2} \\
= \\
=\end{array}$ & $\mid \begin{array}{l}0 \\
0 \\
5 \\
= \\
=\end{array}$ & $\begin{array}{l}\text { in } \\
\text { in } \\
=\end{array}$ & $\begin{array}{l}n \\
+0 \\
\dot{0} \\
= \\
=\end{array}$ & $\begin{array}{l}n \\
? \\
5 \\
= \\
=\end{array}$ & $\begin{array}{l}\infty \\
\stackrel{a}{ } \\
\Xi \\
=\end{array}$ & $\begin{array}{l}9 \\
\stackrel{0}{0} \\
= \\
=\end{array}$ & 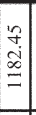 & $\begin{array}{l}\stackrel{n}{i} \\
\stackrel{\infty}{=} \\
=\end{array}$ & $\mid \begin{array}{l}\infty \\
0 \\
0 \\
0 \\
= \\
=\end{array}$ & $\begin{array}{l}\vec{\alpha} \\
\infty \\
\infty \\
=\end{array}$ & 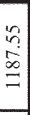 & $\begin{array}{l}\vec{n} \\
2 \\
\dot{\alpha} \\
=\end{array}$ & & $\begin{array}{l}\stackrel{\infty}{a} \\
\frac{a}{2} \\
\stackrel{2}{=}\end{array}$ & & $\mid \begin{array}{l}0 \\
\vdots \\
\vdots \\
ٍ\end{array}$ & \begin{tabular}{l}
$n$ \\
$\vdots$ \\
$\vdots$ \\
$\vdots$ \\
\hdashline
\end{tabular} & 管 & 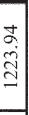 \\
\hline ABUNDANCE per 10 fields & 3 & t & - & 6 & in & $m$ & $?$ & $\overline{0}$ & $\circ$ & $\stackrel{3}{8}$ & $\overrightarrow{0}$ & $\bar{m}$ & $\infty$ & $\stackrel{\infty}{\sim}$ & $\exists$ & $=$ & in & $=$ & m & $m$ & $\infty$ & + & a & + & $\stackrel{\infty}{c}$ \\
\hline Amaurolithus amplificus & & & & & & & & & & & & & & & & & & & & & & & & & \\
\hline A. delicatus & & & & & & & & & & & & & & & & & & & & & & & & & \\
\hline A. primus & & & & & & & & & & & & & & & & & & & & & & & & & \\
\hline A. tricorniculatus & & & & & & & & & & & & & & & & & & & & & & & & & \\
\hline Coccolithus pelagicus & & & & & & & & & & & & & $R$ & & & & & & & & & & & & \\
\hline Catinaster calyculus & & & & & & & & & & & & & & & & & & & & & & & & & \\
\hline C. coalitus & & & & & & & & & & & & & & & & & & & & & & & & & \\
\hline Ceratolithus acutus & & & & & & & & & & & & & & & & & & & & & & & & & \\
\hline C. armatus & & & & & & & & & & & & & & & & & & & & & & & & & \\
\hline C. cristatus & & & & & & & & & & & & & & & & & & & & & & & & & \\
\hline C. rugosus & & & & & & & & & & & & & & & & & & & & & & & & & \\
\hline Coronocyclus nitescens & & & & & & & & & & & & & & & & & & & & & & & & & \\
\hline Cyclicargolithus floridanus & & & & & & & & & & & & P & & $P$ & P & P. & $P$ & P & P... & P & & & & & \\
\hline C. abisectus & & & & & & & & & & & & & & & & & & & & 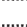 & & & & & \\
\hline Calcidiscus leptoporus & & & & & & & & & & & & & & & & & & & & & & & & & \\
\hline C. macintyrei & & & & & & & & & & & & & & & & & & $R$ & & & & & & & \\
\hline Dictyococcites bisectus & & & & & & & & & & & & & & & & & & & & & & & & & \\
\hline D. productus & & & & & & & & & & & & & & & & & & & & & & & & & \\
\hline Discoaster adamanteus & & & & & & & & & & & & & & & & & & & & & & & & & \\
\hline D. asymmetricus & & & & & & & & & & & & & & & & & & & & & & & & & \\
\hline D. bellus & & & & & & & & & & & & & & & & & & & & & & & & & \\
\hline D. bollii & & & & & & & & & & & & & & & & & & & & & & & & & \\
\hline D. brouweri & & & & & & & .... & & & $\ldots$ & ( & 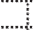 & $\ldots$ & & & & & & & & & & & & \\
\hline D. calcaris & & & & & & & & & & & 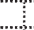 & & & & & & & & & & & & & & \\
\hline D. deflandrei & & & & & & & & & & & 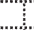 & & & & & & & & & & & & & & \\
\hline D. druggi & & & & & & & & & & & & & & & & & & & & & & & & & \\
\hline D. exilis & & & & & & & & & & & 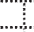 & . & 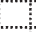 & & & & & & & & & & & & \\
\hline D. hamatus & & & & & & & & & & & . & & 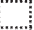 & & & & & & & & & & & & \\
\hline D. neohamatus & & & & & & & & & & & & & & & & & & & & & & & & & \\
\hline D. pentaradiatus & & & & & & & & & & & & & $P$ & & & & . & & & & & & & & \\
\hline D. quinqueramus & & & & & & & & & & & & & & & & & & & & & & & & & \\
\hline D. surculus & & & & & & & & & & & & & & ... & & & 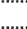 & & & & & & & & \\
\hline D. tamalis & & & & & & & & & & & & & & & & & & & & & & & & & \\
\hline D. variabilis & & & & & & & & & & & & P... & & P & & & P & & & & & & & & \\
\hline Emiliania huxleyi & & & & & & & & & & & & & & & & & & & & & & & & & \\
\hline Ericsonia fenestrata & & & & & & & & & & & & & & & & & & & & & & & & & \\
\hline Gephyrocapsa spp. & . & & & & & & & & & & & $\mathrm{FC}:$ & $F C$ & $\mathrm{FC}$ & $F$ & $F$ & $\mathrm{~F}$ & $F$ & $\mathrm{~F}$ & $F$ & $\mathrm{~F}$ & $\mathrm{~F}$ & $\mathrm{~F}$ & $\mathrm{~F}$ & C. \\
\hline G. oceanica & & & & & & & & & & & & & & & & & & & & & & & & & \\
\hline Helicosphoera ampliaperta & & & & & & & & & & & & & & & & & & & & & & & & . & \\
\hline H. carteri & & & & & & & & & & & & & & & & & & & & & & & & R & R. \\
\hline H. intermedia & & & & & & & & & & & & & & & & & & & & & & & & & \\
\hline H. obliqua & & & & & & & & & & & & & & & & & & & & & & & & & \\
\hline H. sellii & & ma & & . & 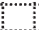 & & . & - & & 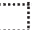 & i & $\mathrm{R}$ & $R$ & $R$ & $R$ & $R$ & $R$ & $R$ & $R$ & $R$ & R & $\mathrm{R}$ & $R$ & R & $\mathrm{R}$ \\
\hline Oolithotus fragilis & & 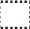 & & & & & & & & & & & & & & & & & & & & & & & 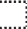 \\
\hline Pseudoemiliania lacunosa & & & & & & & & & & & & & & & & & & & & & & & & & \\
\hline Reticulofenestra spp. & F... & $\mathrm{R}$ & $R$ & $R$ & $\mathrm{R}:$ & $R$ & $R$ & & & $\mathrm{R}$ & $\mathrm{R}$ & $\mathrm{R}$ & F & $R$ & $\mathrm{R}$ & $\mathrm{R}$ & $\mathrm{R}$ & $\mathrm{R}$ & $R$ & R... & R... & $R$ & $F$ & R... & $\mathrm{FC}$ \\
\hline R. minutula & $F$ & R & $R$ & R & $R$ & $R$ & & & & $\mathrm{R}$ & $R:$ & $R$ & & $R$ & $\mathrm{R}$ & $R$ & $\mathrm{R}$ & $\mathrm{R}$ & $R$ & $\mathrm{R}$ & R. & $R$ & $R$ & R & $R$ \\
\hline R. pseudoumbilica & & $\mathrm{R}$ & & & & & & & & & & & & & & & & & & & $\mathrm{R}$ & $R$ & $R$ & & R \\
\hline Rhabdosphaera clavigera & & & & & & & & & & & & & & & & & & & & & & & & & \\
\hline Sphenolithus abies & $R$ & & & & & & & & & & & & & & & & & $R$ & & & & & & & \\
\hline S. belemnos & & & & & & & & & & & & & & & & & & & & & & & & & \\
\hline S. ciperoensis & & & & & & & & & & & & & & & & & & & & & & & & & \\
\hline S. conicus & & & & & & & & & & & & & & & & & & & & & & & & & \\
\hline S. dissimilis & & & & & & & & & & & & & & & & & & & & & & & & & \\
\hline S. distentus & & & & & & & & & & & & & & & & & & & & & & & & & \\
\hline S. heteromorphus & $P$ & $P$ & & & - & & $P$ & & & & & & & $R$ & $R$ & $R$ & $R$ & $R$ & & & & & & & \\
\hline S. moriformis & & & & & & & & & & & & & & & & & & & & & & $R$ & & $R$ & \\
\hline S. neoabies & & & & & $R$ & $R$ & & & & & & & $P$ & & & & & & & & & $\mathrm{R}$ & & & \\
\hline Syracosphaera histrica & & & & & & & & & & & & & & & & & & & & & & & & & \\
\hline Úmbilicosphaera sibogae & & & & & & & 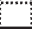 & 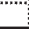 & & & & & & & & & & & & & & & & 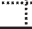 & e \\
\hline
\end{tabular}


Table 6. Nannofossil analysis for 1234.5 - $1366.79 \mathrm{~m}$.

\begin{tabular}{|c|c|c|c|c|c|c|c|c|c|c|c|c|c|c|c|c|c|c|c|c|c|}
\hline 总 & & & & & & & 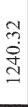 & & & & 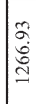 & $\begin{array}{c}\infty \\
\stackrel{+}{0} \\
-\end{array}$ & I্ & 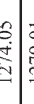 & & & 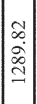 & 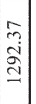 & & लृ: & \\
\hline ABUNDANCE per 10 fields & $\underline{\underline{s}}$ & & $6]$ & 7 & to & & \$ & $\stackrel{2}{\beth}$ & $\stackrel{\circ}{-1}$ & & 2 & $a$ & $m$ & + & & m & -1 & $\sigma$ & $\overrightarrow{0}$ & & \\
\hline Amaurolithus amplificus & & & & & & & & & & & & & & & & & & & & & \\
\hline A. delicatus & & & & & & & & & & & & & & & & & & & & & \\
\hline $\begin{array}{l}\text { A. primus } \\
\text { A. tricorniculatus }\end{array}$ & & & & & & & & & & & & & & & & & & & & & \\
\hline Coccolithus pelagicus & & & & & & & & & & & & & & & & & & R & & R & \\
\hline Catinaster calyculus & & & & & & & & & & & & & & & & & & & & & \\
\hline C. coalitus & & & & & & & & & & & & & & & & & & & & & \\
\hline Ceratolithus acutus & & & & & & & & & & & & & & & & & & $P$ & . & & \\
\hline C. armatus & & & & & & & & & & & & & & & & & & & & & \\
\hline C. cristatus & & & & & & & & & & & & & & & & & & & ....... & & \\
\hline C. rugosus & & & & & & & & & & & & $P$ & & & & & & & & & \\
\hline Coronocyclus nitescens & & & & & & & & & & & & & & & & & & & & & \\
\hline Cyclicargolithus floridanus & & & & & & & & $P$ & P. & & P. & & & & & & & & & ?... & \\
\hline C. abisectus & & & & & & & & & & & & & & & & & & & 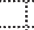 & & \\
\hline Calcidiscus leptoporus & & & & & & & & & & & $R$ & & & & & & R & & & & \\
\hline C. macintyrei & & & & & & & & & & $\mathrm{R}$ & & & & & & & $R$ & & & & \\
\hline Dictyococcites bisectus & & & & & & & & & & & & & & & & & & & .. & & \\
\hline D. productus & & & & & & & & & & & & & & & & & & & & & \\
\hline Discoaster adamanteus & & & & & & & & & & & $\cdots$ & & & & & & & & 米 & & \\
\hline D. asymmetricus & & & & & & & & & & & & & & & & & & & & & \\
\hline D. bellus & & & & & & & & & & & ${ }^{2}$ & & & & & & $\cdots$ & & .. & & \\
\hline $\begin{array}{l}\text { D. bollii } \\
\text { D. brouweri }\end{array}$ & & & & & & & & & & & & & & & & & & & & & \\
\hline D. calcaris & & & & & & & & 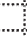 & & & & & & & & & & & 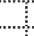 & & \\
\hline D. deflandrei & & & & & & & & & & & & & & & & & & & & & \\
\hline D. druggi & & & & & & & & & & & 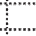 & & & & & & & & .. & & \\
\hline D. exilis & & & & & & & & & & & & & & & & & 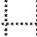 & & ... & & \\
\hline $\begin{array}{l}\text { D. hamatus } \\
\text { D. neohamatus }\end{array}$ & & & & & & & & & & & & & & & & & & & & & \\
\hline $\begin{array}{l}\text { D. neohamatus } \\
\text { D. pentaradiatus }\end{array}$ & $R$ & & R & $R$ & R & $R$ & $R$ & R & $R$ & & $R$ & & & & & & & & & ? & \\
\hline D. quinqueramus & & & & & & & & & & & & & & & & & & & & & \\
\hline D. surculus & & & & & & & & & & & & & & & & $\ldots .$. & $\xi_{\ldots}$ & & $\ldots$ & & \\
\hline D. tamalis & & & & & & & & & & & & & & & & & & & & & \\
\hline D. variabilis & & & & & & & & & & & & & & & & & & & & & \\
\hline Emiliania huxleyi & & & & & & & & & & & & & & & & & & & & & \\
\hline Ericsonia fenestrata & & & & & & & & & & & & & & & & & & & & & \\
\hline Gephyrocapsa spp. & c & & & & & & C. & c.. & c. & C & $c$ & & & & & R. & R. & & & ?a & $R$ \\
\hline G. oceanica & & & & & & & $u^{\prime}$ & me & & & & & & & & & ....... & & & & \\
\hline Helicosphoera ampliaperta & & & & & & & & & & & & & & & & & & & & & \\
\hline H. carteri & R. & & Re & $\mathrm{R}$ & $\mathrm{R}$ & $R$ & $\mathrm{R}$ & $R$ & $R:$ & $\underline{\mathrm{R}}$ & 8 & & & & & & R. & & & $\mathrm{R}$ & \\
\hline H. intermedia & ........ & & & & & & & - & & & 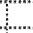 & $x_{0}$ & & & & & & ....... & ... & & \\
\hline H. obliqua & & & & & & & & & & & & & & & & & & & & & \\
\hline $\begin{array}{l}\text { H. sellii } \\
\text { Oolithotus fragilis }\end{array}$ & & & & & & & & & & & $\vdots$ & & & & & & & & & & \\
\hline Pseudoemiliania lacunosa & & & & & & & & & & & & & & & & & & & & & \\
\hline Reticulofenestra spp. & & & & & & & & & & & EC & & & & & & & & & & \\
\hline R. minutula & & & Rin & $\mathrm{R}$ & & & $\mathrm{R}$ & & $R=$ & & $R$ & & & & & & & & & F & \\
\hline R. pseudoumbilica & & & & & & & & & & & $\mathrm{R}$ & & & & & & & $P$ & & 5 & $\mathrm{VF}$ \\
\hline Rhabdosphaera clavigera & & & & & & & & & & & & & & & & & & & & & \\
\hline Sphenolithus abies & & & $P$ & $\mathrm{P}$ & $P$ & & $P$ & $P$ & $P:$ & & $P$ & & & & & & & & & & \\
\hline S. belemnos & & & & & & & & & & & & & & & & 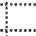 & & & 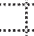 & & \\
\hline S. ciperoensis & & & & & & & & & & & & & & & & & & & & & \\
\hline S. conicus & & & & & & & & & & & & & & & & & & & & & \\
\hline S. dissimilis & & & & & & & & & & & & & & & & & & & & & \\
\hline S. distentus & & & & & & & & & & & & & & & & & & & & & \\
\hline S. heteromorphus & & & & $\mathrm{P}$ & $P$ & & & & & & & & & & & & & & & & $\ldots$ \\
\hline S. moriformis & R. & & & & & & & & & & & & & & & & & & & & \\
\hline S. neoabies & & & $R$ & & & & $\mathrm{R}$ & R. & & & $R$ & & & & & & $R$ & & & P... & \\
\hline Syracosphaera histrica & & & & & & & & & & & & & & & & & & & & & \\
\hline Umbilicosphaera sibogae & & & a & & & & & $\cdots$ & $\cdots$ & & 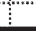 & & & & & 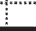 & $I^{\prime \prime}$ & ‥' & 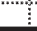 & & 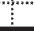 \\
\hline
\end{tabular}


Table 7. Nannofossil analysis for $1378.41-1550.83 \mathrm{~m}$.

\begin{tabular}{|c|c|c|c|c|c|c|c|c|c|c|c|c|c|c|c|c|c|c|c|c|c|c|c|c|c|}
\hline TCDP-A & $\begin{array}{l}\overrightarrow{3} \\
\overrightarrow{3} \\
\hat{m}\end{array}$ & $\mid$ & $\begin{array}{l}\overrightarrow{7} \\
\hat{n} \\
\hat{2}\end{array}$ & 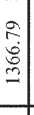 & $\mid$\begin{tabular}{|c}
$\vec{\sigma}$ \\
$\infty$ \\
0 \\
0 \\
-2
\end{tabular} & 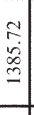 & $\begin{array}{l}\infty \\
\stackrel{2}{2} \\
2 \\
-2\end{array}$ & $\mid \begin{array}{l}0 \\
0 \\
\dot{0} \\
\vdots \\
\end{array}$ & 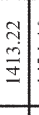 & 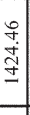 & 志 & $\stackrel{9}{ \pm}$ & \begin{tabular}{l}
$\infty$ \\
$\vdots$ \\
$\dot{f}$ \\
\hdashline
\end{tabular} & $\begin{array}{l}0 \\
0 \\
\vdots \\
-\end{array}$ & 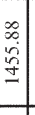 & 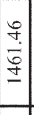 & $\begin{array}{l}3 \\
3 \\
5 \\
0 \\
0\end{array}$ & 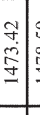 & $\begin{array}{l}0 \\
n \\
\alpha \\
\alpha \\
v \\
-\end{array}$ & 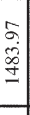 & $\left|\begin{array}{l}0 \\
0 \\
\vdots \\
0 \\
\vdots \\
- \\
-1\end{array}\right|$ & 哭 & $\begin{array}{l}2 \\
8 \\
8 \\
2\end{array}$ & 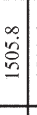 & 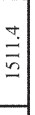 \\
\hline ABUNDANCE per 10 fields & - & 0 & $\infty$ & 0 & 0 & + & 0 & 0 & $\overrightarrow{0}$ & $\overrightarrow{0}$ & 0 & + & $\stackrel{3}{0}$ & 0 & 0 & $\circ$ & 0 & ฮิ) & $\stackrel{1}{\circ}$ & 0 & ֻั) & - & 0 & 0 & $?$ \\
\hline Amaurolithus amplificus & & & & & & & & & & & & & & & & & & & & & & & & & \\
\hline A. delicatus & & & & & & & & & & & & & & & & & & & & & & & & & \\
\hline A. primus & & & & & & & & & & & & & & & & & & & & & & & & & \\
\hline A. tricorniculatus & & & & & & & & & & & & & & & & & & & & & & & & & \\
\hline Coccolithus pelagicus & & $R$ & $R$ & & & & & & & & & & P... & & & & & & & & & & & & \\
\hline Catinaster calyculus & & & & & & & & & & & & & & & & & & & & & & & & & \\
\hline C. coalitus & & & & & & & & & & & & & & & & & & & & & & & & & \\
\hline Ceratolithus acutus & & & & & & & & & & & & & & & & & & & & & & .... & & & \\
\hline C. armatus & & & & & & & & & & & & & & & & & & & & & & & & & \\
\hline C. cristatus & & & & & & & & & & & & & ........ & & & & & & & & & & & & ....... \\
\hline C. rugosus & & & & & & & & & & & & & & & & & & 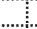 & & & & .. & & & .... \\
\hline Coronocyclus nitescens & & & & & & & & & & & & & & & & & & & & & & & & & \\
\hline Cyclicargolithus floridanus & & & & & & & & & & & & & & & & & & & & & & & & & \\
\hline C. abisectus & & & & & & & & & & & & & & & & & & & & & & & & & , \\
\hline Calcidiscus leptoporus & & & & & & & & & & & & & & & & & & & & & & P... & & & 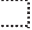 \\
\hline C. macintyrei & & & & & & & & & & & & & & P..... & & & & & & & & & & & \\
\hline Dictyococcites bisectus & & & & & & & & & & & & & & & & & & & & & & & & & \\
\hline D. productus & & & & & & & & & & & & & $P$ & & & & & & & & & $P$ & & & 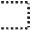 \\
\hline Discoaster adamanteus & & & & & & & & & & & & & & & & & & & & & & & & & .... \\
\hline D. asymmetricus & & & & & & & & & & & & & & & & & & & & & & & & & \\
\hline D. bellus & & & & & & & & & & & & & & & & & & & & & & & & & \\
\hline D. bollii & & & & & & & & & & & & & & & & & & & & & & & & & \\
\hline D. brouweri & & & & & & & & & & & & & & $P$ & & & & & & & & & & & \\
\hline D. calcaris & & & & & & & & & & & & & & & & & & & & & & & & & \\
\hline D. deflandrei & & & & & & & & & & & & & & & & & & $P$ & & & & & & & \\
\hline D. druggi & & & & & & & & & & & & & & & & & & & & & & & & & \\
\hline D. exilis & & & & & & & & & & & & & & & & & & & & & & & & & \\
\hline D. hamatus & & & & & & & & & & & & & & & & & & & & & & & & & \\
\hline D. neohamatus & & & & & & & & & & & & & & & & & & & & & & & & & \\
\hline D. pentaradiatus & & $P$ & & & & & & & & & & & & & & & & & & & & & & & 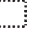 \\
\hline D. quinqueramus & & & & & & & & & & & & & & & & & & & & & & & & & \\
\hline D. surculus & & & & & & & & & & & & & & & & & & & & & & & & & \\
\hline D. tamalis & & $P$ & & & & & & & & & & & & & & & & & & & & & & & \\
\hline D. variabilis & & & & & & & & & & & & & & & & & & & & & & $P$ & & & 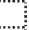 \\
\hline Emiliania huxleyi & & & & & & & & & & & & & & & & & & & & & & & & & \\
\hline Ericsonia fenestrata & & & & & & & & & & & & & & & & & & & & & & & & & \\
\hline Gephyrocapsa spp. & & $R$ & R & & & $R$ & & & $\mathrm{P}$ & $P$ & & $R$ & & $R$ & & & & $P$ & $P$ & $R$ & & $R$ & & & 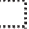 \\
\hline G. oceanica & & & & & & & & & & & & & & & & & & & & & & & & & $\cdots$ \\
\hline Helicosphoera ampliaperta & & & & & & & & & & & & & & & & & & & & & & & & & \\
\hline H. carteri & & & & & & & & & & & & & & & & & & & & & & & & & \\
\hline H. intermedia & & & & & & & & & & & & & & & & & & & & & & & & & \\
\hline H. obliqua & & & & & & & & & & & & & & & & & & & & & & & & & ..... \\
\hline H. sellii & & $R$ & $R$ & & & & & & & & & & & & & & & & & & & P. & & & \\
\hline Oolithotus fragilis & & & & & & & & & & & & & & & & & & & & & & & & & \\
\hline Pseudoemiliania lacunosa & & $R:$ & VR: & & & & & & & & & $P$ & & & & & & P. & $\mathrm{P}$ & $R$ & & & & & 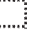 \\
\hline Reticulofenestra spp. & & $R$ & $R$ & & & & & & & & & & & $R$ & & & & p" & & & & $R$ & & & $\mathrm{R}$ \\
\hline R. minutula & & $P$ & & & & & & & & & & & & & & & & & & & & & & & \\
\hline R. pseudoumbilica & & & & & & & & & & & & & & & & & & & & & & & & & \\
\hline Rhabdosphaera clavigera & & & & & & & & & & & & & & & & & & & & & & & & & \\
\hline Sphenolithus abies & & & $P$ & & & & & & & & & & & & & & & & & & & & & & \\
\hline S. belemnos & & & & & & & & & & & & & & & & & & & & & & & & & \\
\hline S. ciperoensis & & & & & & & & & & & & & & & & & & & & & & & & & \\
\hline S. conicus & & & & & & & & & & & & & & & & & & & & & & & & & ..... \\
\hline S. dissimilis & & & & & & & & & & & & & & & & & & & & & & & & & ....... \\
\hline S. distentus & & & & & & & & & & & & & & & & & & & & & & & & & \\
\hline S. heteromorphus & & & & & & & & & & & & & & & & & & & & & & & & & \\
\hline S. moriformis & & & & & & & & & & & & & & & & & & & & & & & & & \\
\hline S. neoabies & & & & & & & & & & & & & & & & & & & & & & $P$ & & & 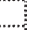 \\
\hline Syracosphaera histrica & & & & & & & & & & & & & & & & & & & & & & & & & \\
\hline Umbilicosphaera sibogae & & & & & & & & & & & & & & & & & & & & & & & & & $\cdots$ \\
\hline
\end{tabular}


Table 8. Nannofossil analysis for $1556.48-1728.3 \mathrm{~m}$.

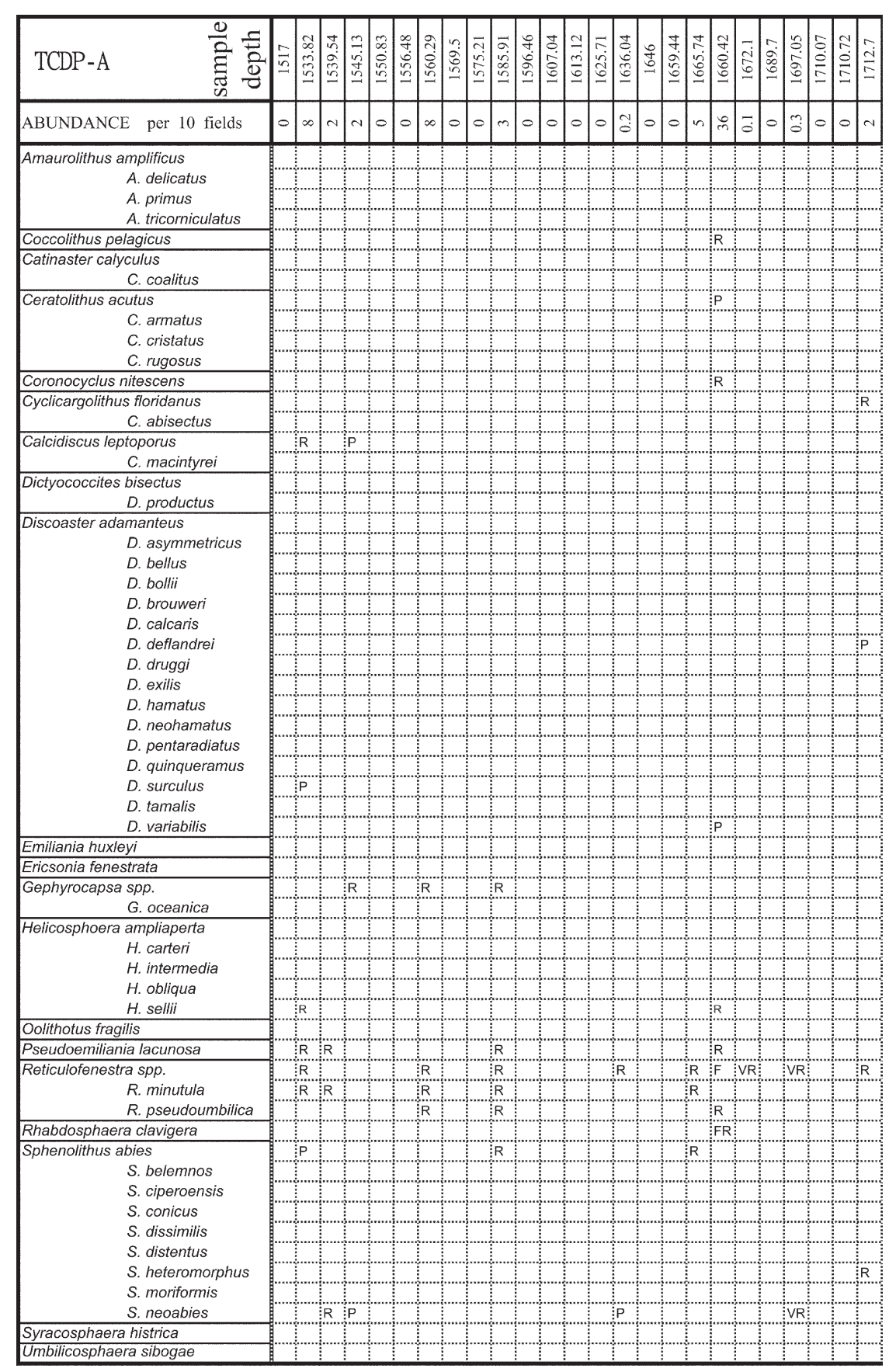


Table 9. Nannofossil analysis for 1748.8 - $1938.49 \mathrm{~m}$.

\begin{tabular}{|c|c|c|c|c|c|c|c|c|c|c|c|c|c|c|c|c|c|c|c|c|c|c|c|c|c|}
\hline TCDP-A & $\underset{\underline{\Xi}}{\stackrel{\infty}{ \pm}}$ & $\begin{array}{l}\stackrel{\infty}{\infty} \\
\stackrel{\infty}{\Xi}\end{array}$ & 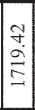 & 足 & 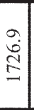 & 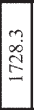 & $\begin{array}{l}\infty \\
\infty \\
0 \\
\vdots \\
\underline{I}\end{array}$ & 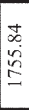 & $\begin{array}{l}+ \\
\infty \\
\stackrel{0}{E} \\
=\end{array}$ & $\begin{array}{l}2 \\
\text { s. } \\
2 \\
2\end{array}$ & $\mid \begin{array}{l}0 \\
2 \\
\vdots \\
0 \\
-0\end{array}$ & $\underset{\vec{D}}{\vec{\Xi}}$ & $\begin{array}{l}\hat{\overrightarrow{3}} \\
\stackrel{\infty}{-}\end{array}$ & $\left|\begin{array}{c}m \\
\stackrel{2}{2} \\
\underline{0}\end{array}\right|$ & $\begin{array}{c}3 \\
\vdots \\
\vdots \\
\infty \\
-\end{array}$ & $\begin{array}{l}\tilde{0} \\
\dot{+} \\
\underline{\infty}\end{array}$ & 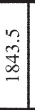 & $\begin{array}{l}\overrightarrow{2} \\
2 \\
\approx \\
-\end{array}$ & & 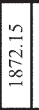 & $\mid \begin{array}{c}1 \\
5 \\
2 \\
2 \\
0 \\
-1\end{array}$ & 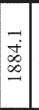 & $\begin{array}{l}n \\
n \\
\infty \\
\infty \\
\infty \\
\infty \\
-1\end{array}$ & $\begin{array}{l}= \\
\overrightarrow{0} \\
\infty \\
\infty \\
\infty\end{array}$ & \\
\hline ABUNDANCE per 10 fields & n & 0 & 0 & - & - & $\because$ & o & 0 & $\because$ & $\overline{0}$ & 0 & ? & 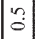 & 0 & o & $\circ$ & 0 & $\circ$ & 0 & 0 & $\sim$ & - & - & $\stackrel{m}{0}$ & - \\
\hline \multicolumn{26}{|l|}{ Amaurolithus amplificus } \\
\hline A. delicatus & & & & & & & & & & & & & & & & & & & & & & & & & \\
\hline A. primus & & & & & & & & & & & & & & & & & & & & & & & & & \\
\hline A. tricorniculatus & & & & & & & & & & & & & & & & & & & & & & & & & \\
\hline \multicolumn{26}{|l|}{ Coccolithus pelagicus } \\
\hline \multicolumn{26}{|l|}{ Catinaster calyculus } \\
\hline C. coalitus & & & & & & & & & & & & & & & & & & & & & & & & & \\
\hline \multicolumn{26}{|l|}{ Ceratolithus acutus } \\
\hline C. armatus & & & & & & & & & & & & & & & & & & & & & & & & & \\
\hline C. cristatus & & & & & & & & & & & & & & & & & & & & & ... & & & & \\
\hline C. rugosus & & & & & & & & & & & & & & & & & & & & & & & & & \\
\hline \multicolumn{26}{|l|}{ Coronocyclus nitescens } \\
\hline Cyclicargolithus floridanus & & & & $R$ & & $P$ & & & $R$ & & & $R$ & P & & & & & & & & $P$ & P & R & $\mathrm{P}$ & $R$ \\
\hline C. abisectus & & & & & & & & & & & & & & & & & & & & & & & & & \\
\hline Calcidiscus leptoporus & & & & & & P.. & & & & & & & & & & & & & & & & & & & \\
\hline C. macintyrei & & & & & & & & & & & & & & & & & & & & & . & & & & \\
\hline Dictyococcites bisectus & & & & & & & & & & & & & & & & & & & & & .... & & & & \\
\hline D. productus & & & & & & & & & & & & & & & & & & & & & & & & & $\cdots$ \\
\hline Discoaster adamanteus & & & & & & & & & & & & & & & & & & & & & & & & & \\
\hline D. asymmetricus & & & & 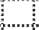 & & & & & & & & & & & & & & & & & ..... & ... & & $\ldots$ & \\
\hline D. bellus & & & & & & & & & & & & & & & & & & & & & & & & .... & \\
\hline D. bollii & & & & & & & & & & & & & & & & & & & & & & & & a & \\
\hline D. brouweri & & & & & & & & & & & & & & & & & & & & & & & & & \\
\hline D. calcaris & & & & & & & & & & & & & & & & & & & & & 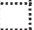 & & & & \\
\hline D. deflandrei & & & & & & P & & & & & & & & & & & & & & & ...... & & & & \\
\hline D. druggi & & & & & & & & & & & & & & & & & & & & & ....... & & & & \\
\hline D. exilis & & & & & & & & & & & & & & & & & & & & & & & & & \\
\hline D. hamatus & & & & & & & & & & & & & & & & & & & & & 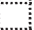 & & & & \\
\hline D. neohamatus & & & & & & & & & & & & & & & & & & & & & $\ldots$ & & & & \\
\hline D. pentaradiatus & & & & & & & & & & & & & & & & & & & & & $\cdots$ & & & & \\
\hline D. quinqueramus & & & & & & & & & & & & & & & & & & & & & ....... & & & & \\
\hline D. surculus & & & & & & & & & & & & & & & & & & & & & & & & & \\
\hline D. tamalis & & & & & & & & & & & & & & & & & & & & & & & & & \\
\hline D. variabilis & & & & & & & & & $\mathrm{VR}$ & & & & $P$ & & & & & & & & $p$ & & P. & & \\
\hline Emiliania huxleyi & & & & & & & & & & & & & & & & & & & & & & & & & \\
\hline Ericsonia fenestrata & & & & & & & & & & & & & & & & & & & & & & & & & \\
\hline Gephyrocapsa spp. & & & & $P$ & & & & & & & & & & & & & & & & & $R$ & $\mathrm{R}$ & $\mathrm{R}$ & ... & $\cdots \cdots$ \\
\hline $\begin{array}{r}\text { G. oceanica } \\
\end{array}$ & & & & & & & & & & & & & & & & & & & & & & & & & \\
\hline Helicosphoera ampliaperta & & & & & & & & & & & & & & & & & & & & & & & & & \\
\hline H. carteri & & & & & & & & & & & & & & & & & & & & & & & & & \\
\hline H. intermedia & & & & 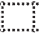 & & & & & & & & & & & & & & & & & $\cdots$ & & & & \\
\hline H. obliqua & & & & & & & & & & & & & & & & & & & & & 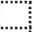 & & & & \\
\hline H. sellii & & & & $P$ & & & & & & & & & & & & & & & & & & 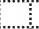 & & & \\
\hline Oolithotus fragilis & & & & & & & & & & & & & & & & & & & & & . & & & & \\
\hline Pseudoemiliania lacunosa & & & & $P$ & & & & & & & & & $P$ & & & & & & & & & & & & \\
\hline Reticulofenestra spp. & & & & & & $P$ & & & $R$ & & & $R$ & $R$ & & & & & & & & $R$ & $R$ & & $P:$ & $R$ \\
\hline R. minutula & & & & Re & & & & & & & & & & & & & & & & & R & & & & \\
\hline R.pseudoumbilica & & & & & & & & & & & & & & & & & & & & & $R$ & $\mathrm{R}$ & P... & & \\
\hline Rhabdosphaera clavigera & & & & & & & & & & & & & & & & & & & & & & & & & \\
\hline Sphenolithus abies & & & & & & & & & & & & & & & & & & & & & & & & & \\
\hline S. belemnos & & & & & & & & & & & & & & & & & & & & & & & & & \\
\hline S. ciperoensis & & & & & & & & & & & & & & & & & & & & & & & & & \\
\hline S. conicus & & & & $R$ & & & & & & & & & & & & & & & & & ..... & & & & \\
\hline S. dissimilis & & & & & & & & & & & & & & & & & & & & & & & & & \\
\hline S. distentus & & & & & & & & & & & & & & & & & & & & & & & & & \\
\hline S. heteromorphus & & & & & & & & & $R$ & & & $P$ & & & & & & & & & $R$ & $P$ & & & $P$ \\
\hline S. moriformis & & & & $R$ & & P. & & & & & & & & & & & & & & & & & & & \\
\hline S. neoabies & & & & & & & & & & & & & & & & & & & & & & & & & \\
\hline Syracosphaera histrica & & & & & & & & & & & & & & & & & & & & & & & & & \\
\hline Umbilicosphaera sibogae & & & & $P$ & & & & & & & & & & & & & & & & & & 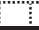 & & & \\
\hline
\end{tabular}


Table 10. Nannofossil analysis for 1939.52 - 2003 m.

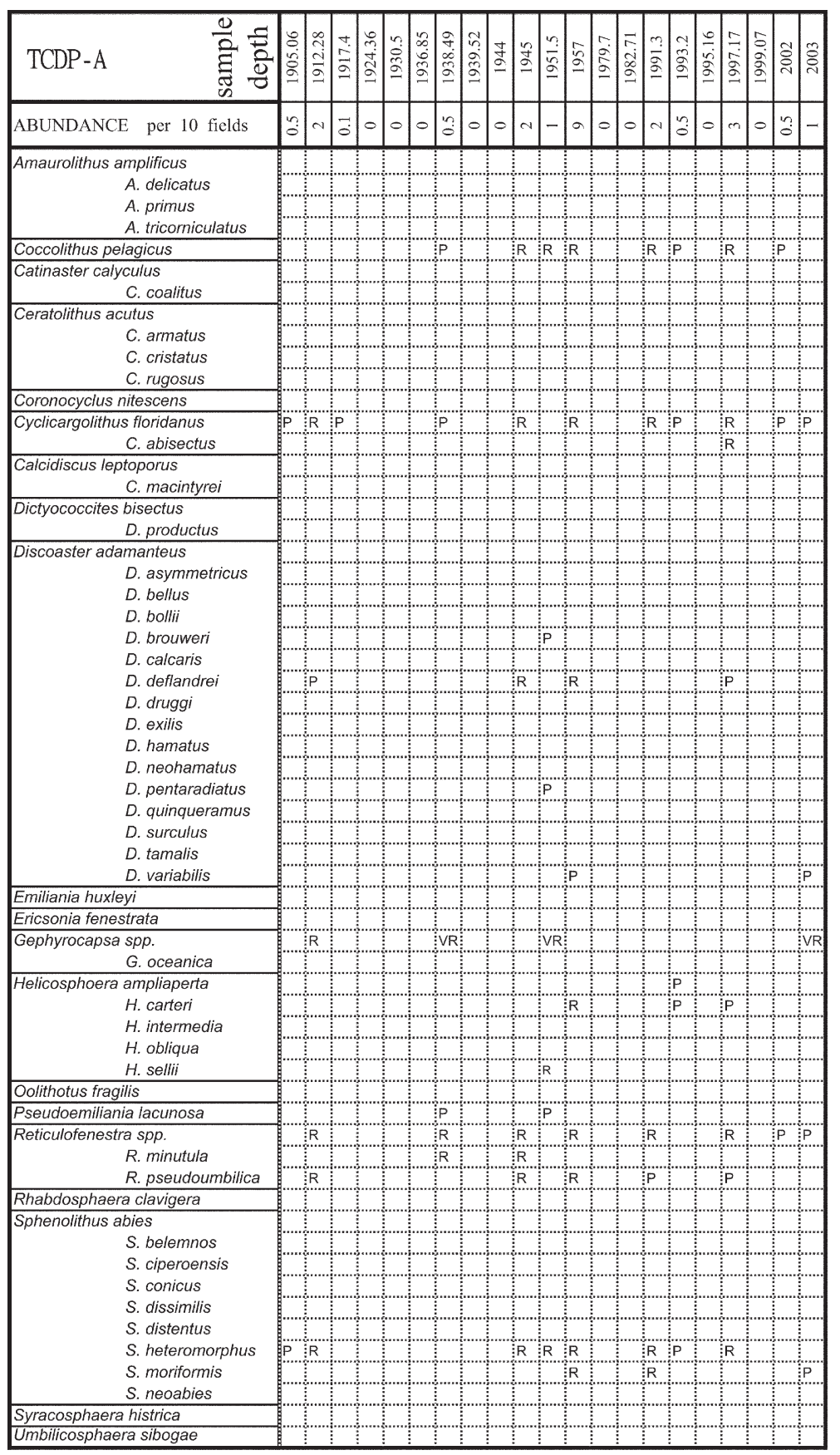




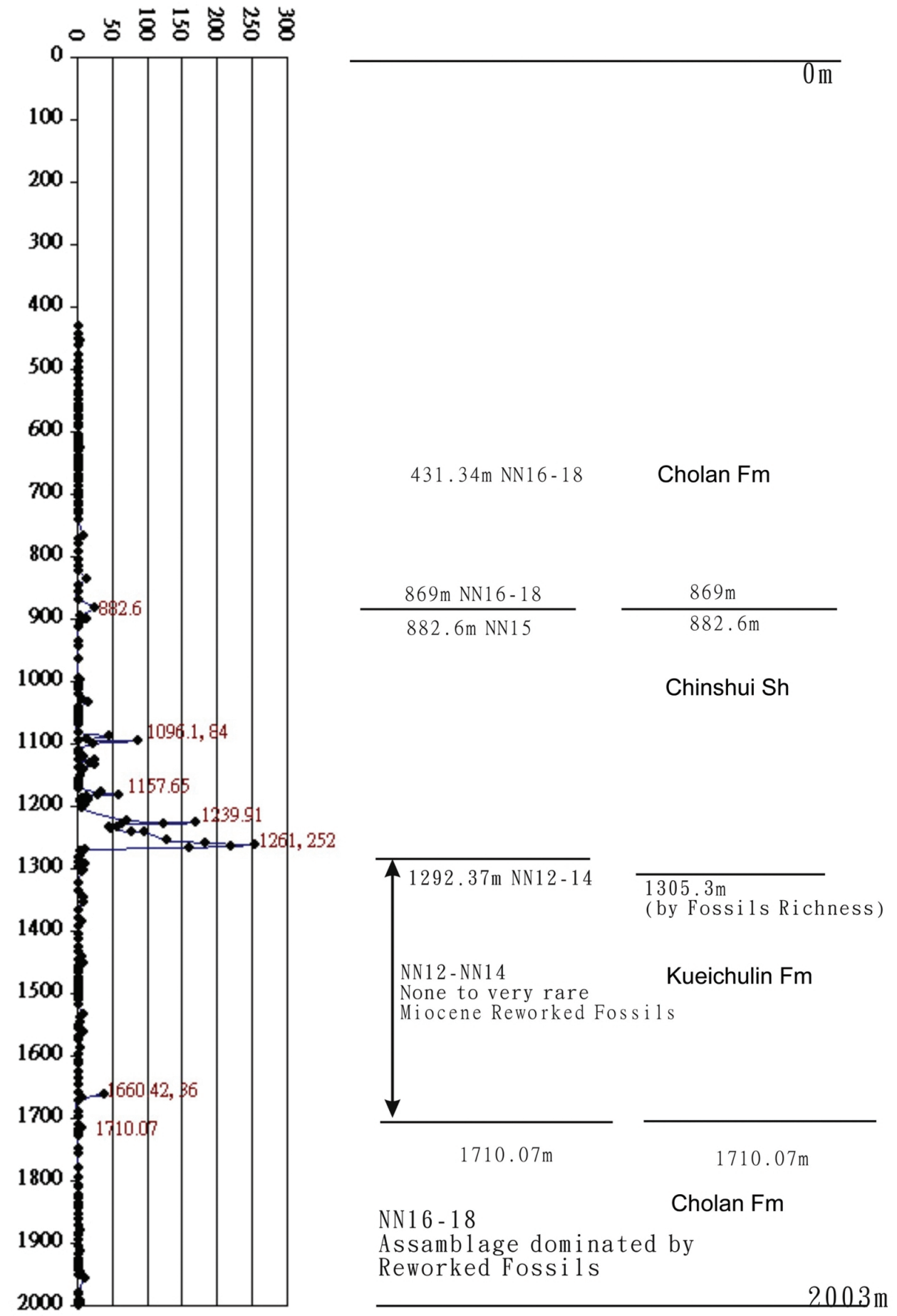

Fig. 6. Nanofossil zoning and richness distribution in the TCDP well-A. 
(a)

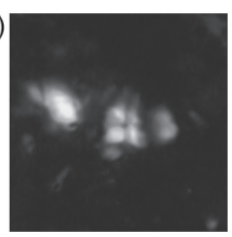

(d)

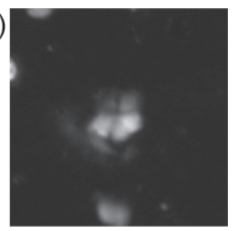

(b)

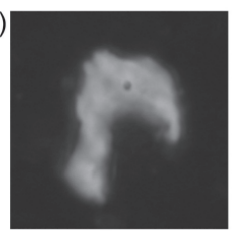

(e)

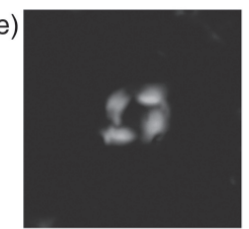

(c)

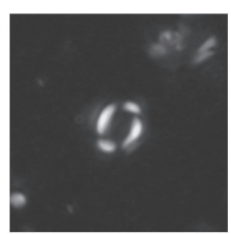

(f)

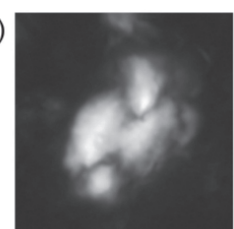

Fig. 7. The nannofossil plate at $1092.6 \mathrm{~m}$ in TCDP-A. (a) Sphenolithus neoabies Bukry \& Bramlette: TCDP-A, $1092.6 \mathrm{~m}, \times 1250$, cross-polarized; (b) Ceratolithus sp.: TCDP-A, $1092.6 \mathrm{~m}, \times 1250$, cross-polarized; (c) Gephyrocapsa sp. Kamptner: TCDP-A, $1092.6 \mathrm{~m}, \times 1250$, crosspolarized; (d) Braarudosphaera bigelowii Deflandre: TCDP-A, $1092.6 \mathrm{~m}$, $\times 1250$, cross-polarized; (e) Reticulofenestra pseudoumbilica Gartner: TCDP-A, 1092.6 m, × 1250, cross-polarized; (f) Helicosphaera kamptneri Hay \& Mohler in Hay et al.: TCDP-A, 1092.6 m, × 1250, cross-polarized.

(a)

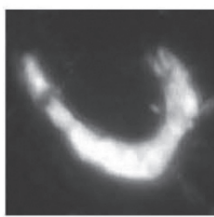

(d)

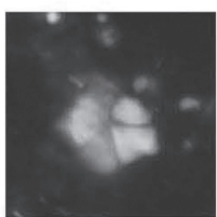

(b)

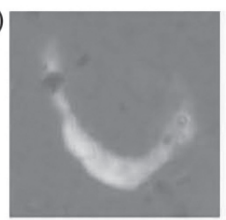

(e)

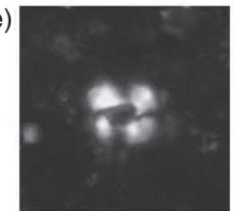

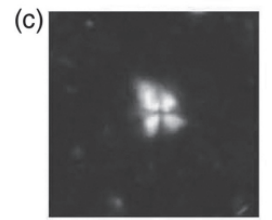

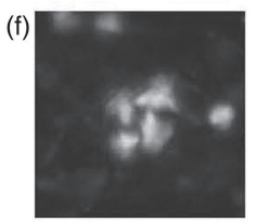

Fig. 8. The nannofossil plate at $1110.25 \mathrm{~m}$ in TCDP-A. (a) Ceratolithus sp., Kamptner (1950): TCDP-A, $1110.25 \mathrm{~m}, \times 1250$, cross nicol; (b) Ceratolithus sp. Kamptner (1950): TCDP-A, 1110.25 m, $\times 1250$, interferencecross; (c) Sphenolithus neoabies Bukry \& Bramlette (1969a): TCDP-A, $1110.25 \mathrm{~m}, \times 1250$, cross nicol; (d) Braarudosphaera bigelowii Deflandre (1947): TCDP-A, $1092.6 \mathrm{~m}, \times 1250$, cross nicol; (e) Reticulofenestra pseudoumbilica Gartner (1969c): TCDP-A, 1110.25 m, $\times 1250$, cross nicol; (f) Reticulofenestra pseudoumbilica Gartner (1969c): TCDP-A, $1110.25 \mathrm{~m}, \times 1250$, cross nicol. 
(a)

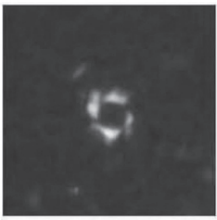

(d)

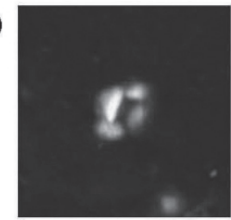

(b)

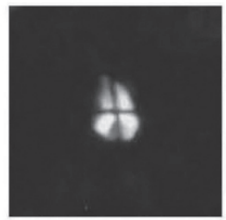

(e)

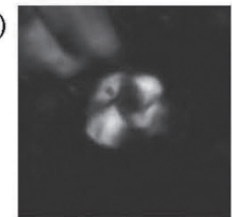

(c)

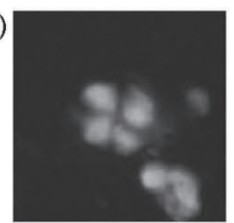

(f)

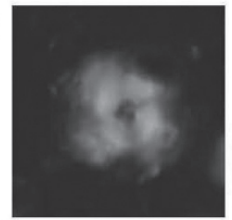

Fig. 9. The nannofossil plate at $1189.51 \mathrm{~m}$ in TCDP-A. (a) Reticulofenestra sp. Kamptner 1943: TCDP-A, $1189.51 \mathrm{~m}, \times 1250$, cross nicol; (b) Sphenolithus abies Deflandre in Deflandren \& Fert (1954): TCDP-A, $1189.51 \mathrm{~m}, \times 1250$, cross nicol; (c) Calcidiscus leptoporus Loeblich \& Tappan (1978): TCDP-A, $1189.51 \mathrm{~m}, \times 1250$, cross nicol; (d) Reticulofenestra minutula Haq \& Berggren (1978): TCDP-A, 1189.51 m, $\times 1250$, cross nicol; (e) Reticulofenestra pseudoumbilica Gartner (1969c): TCDP-A, $1189.51 \mathrm{~m}, \times 1250$, cross nicol; (f) Calcidiscus macintyrei Loeblich \& Tappan (1978): TCDP-A, $1189.51 \mathrm{~m}, \times 1250$, cross nicol.

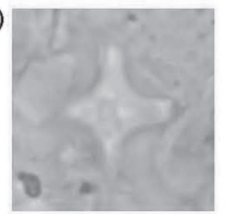

(d)

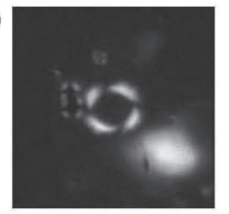

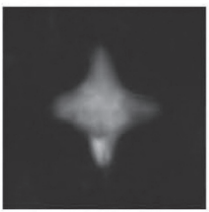

(e)

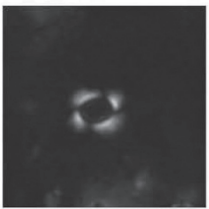

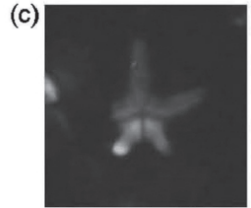

(f)

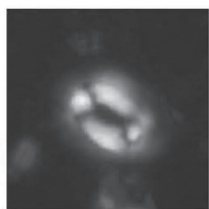

Fig. 10. The nannofossil plate of $1346.35 \mathrm{~m}$ in TCDP-A. (a) Discoaster tamalis Kamptner (1967): TCDP-A, 1346.35 m, × 1250, interference; (b) Discoaster tamalis Kamptner (1967): TCDP-A, 1346.35 m, $\times 1250$, cross nicol; (c) Discoaster pentaradiatus Bramlette \& Riedel (1954): TCDPA, $1346.35 \mathrm{~m}, \times 1250$, interference; (d) Pseudoemiliania lacunosa Gartner (1969c): TCDP-A, $1346.35 \mathrm{~m}, \times 1250$, cross nicol; (e) Reticulofenestra minutula Haq \& Berggren (1978): TCDP-A, $1346.35 \mathrm{~m}, \times 1250$, cross nicol; (f) Coccolithus pelagicus Schiller (1930): TCDP-A, 1346.35 m, $\times 1250$, cross nicol. 
(a)

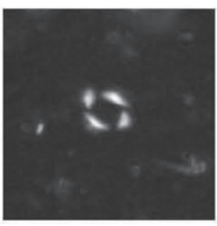

(d)

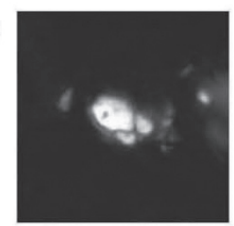

(b)

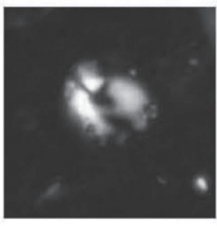

(e)

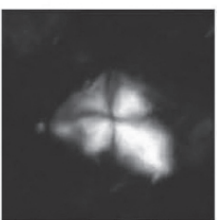

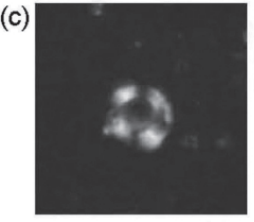

(f)

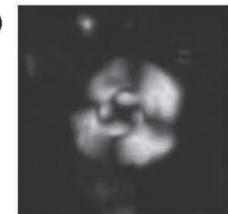

Fig. 11. The nannofossil plate at $1723.7 \mathrm{~m}$ in TCDP-A. (a) Pseudoemiliania lacunosa Gartner (1969c): TCDP-A, $1723.7 \mathrm{~m}, \times 1250$, cross nicol; (b) Helicosphaera sellii Bukry \& Bramlette (1969b): TCDP-A, $1723.7 \mathrm{~m}$, $\times 1250$, cross nicol; (c) Umbilicosphaera sibogae Garder (1970): TCDP-A, $1723.7 \mathrm{~m}, \times 1250$, interference; (d) Sphenolithus heteromorphus deflandre (1953): TCDP-A, $1723.7 \mathrm{~m}, \times 1250$, cross nicol; (e) Sphenolithus conicus Bukry (1971): TCDP-A, $1723.7 \mathrm{~m}, \times 1250$, cross nicol; (f) Cyclicargolithus floridanus Bukry (1971a): TCDP-A, $1723.7 \mathrm{~m}, \times 1250$, cross nicol.

(a)

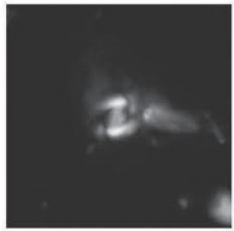

(d)

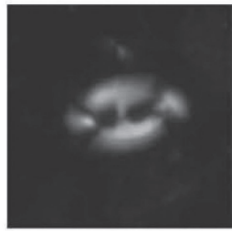

(b)

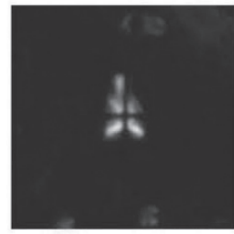

(e)

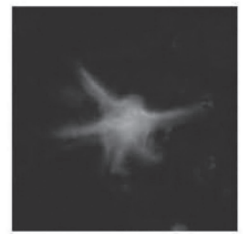

(c)

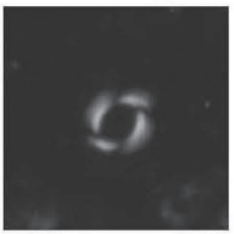

(f)

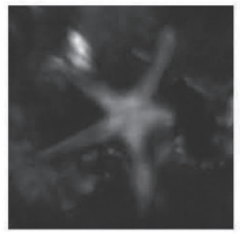

Fig. 12. The nannofossil plate at $1951.5 \mathrm{~m}$ in TCDP-A. (a) Gephyrocapsa sp. Kamptner (1943): TCDP-A, 1951.5 m, $\times 1250$, interference; (b) Sphenolithus abies Deflandre in Deflandren \& Fert (1954): TCDP-A, 1951.5 m, × 1250, cross nicol; (c) Reticulofenestra minutula Haq \& Berggren (1978): TCDP-A, $1951.5 \mathrm{~m}, \times 1250$, interference; (d) Helicosphaera sellii Bukry \& Bramlette (1969b): TCDP-A, 1951.5 m, $\times 1250$, cross nicol; (e) Discoaster brouweri Bramlette \& Riedel (1954): TCDP-A, $1951.5 \mathrm{~m}, \times 1250$, cross nicol; (f) Discoaster pentaradiatus Bramlette \& Riedel (1954): TCDP-A, $1951.5 \mathrm{~m}, \times 1250$, interference. 
and difficult to subdivide, even though they exist in central and northwestern Taiwan (Table 11). Generally, to assist in making biostratigraphic analysis for the CPC exploration, the boundary between NN14 and 15 is used as criteria for both the sharp extinction of the large fossil Reticulofenestra pseudoumbilica and the sharp decrease of Sphenolithus abies. But through out the study area Reticulofenestra pseudoumbilica (large type) tends to be migratable and the distribution of Sphenolithus abies is not very uniform in central and northern Taiwan, making it difficult to accurately distinguish the boundary between NN14 and 15. Therefore, determination of the stratigraphic boundary between the Kuichulin and Chinshui Shales requires lithological analysis data.

In order to constrain the nannofossil index for the subsurface fossil analysis of TCDP well-A,

Table 11. Stratigraphy in central and northern Taiwan (Revised from Chi 1981; Huang 1982; Wu et al. 2002).

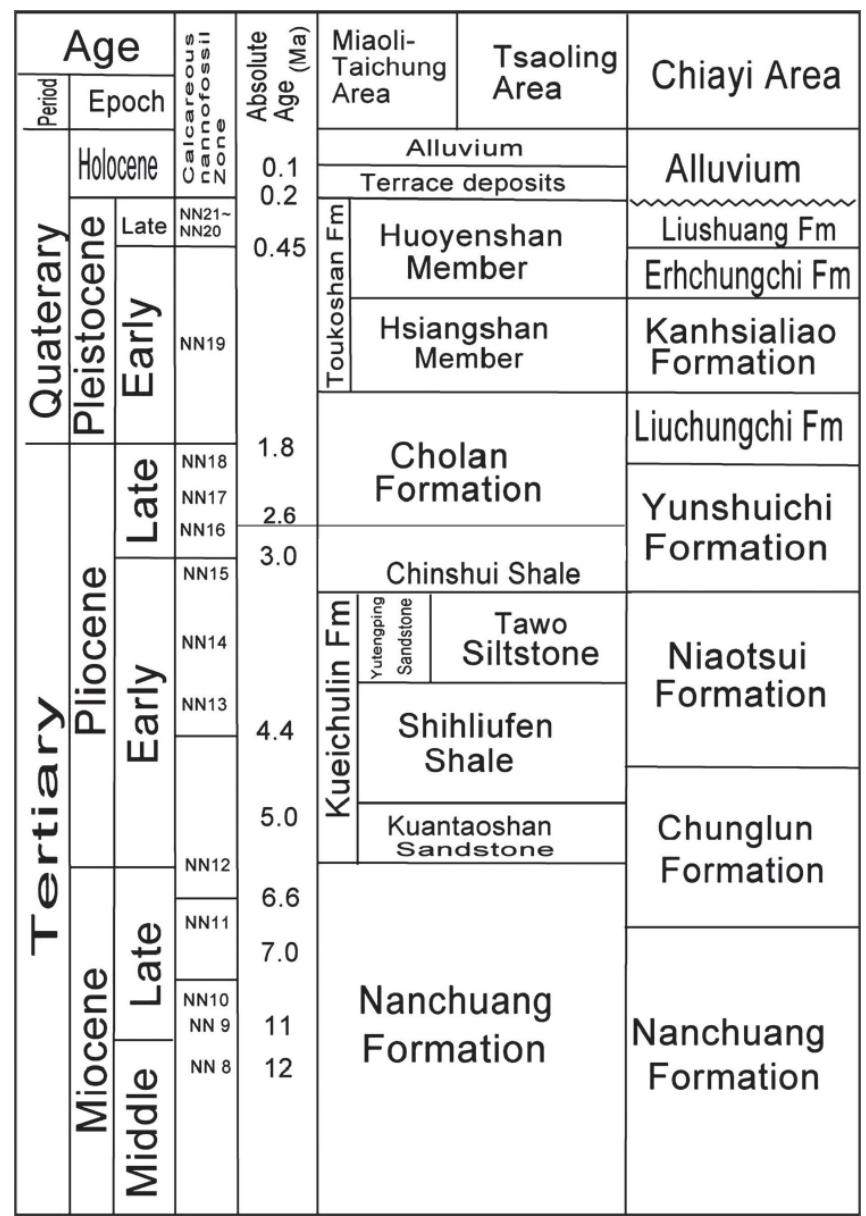


9 samples were taken from an outcrop near this well (Fig. 14). The analysis results are shown in Table 12, where sample no.1 is rare among fossils but whose fossil assemblage is possibly of the Pliocene age. Samples nos. 2 and 3 are NN15, equivalent to the Chinshui Shale; Sample no. 4 is NN12 - 13, equivalent to the lower part of the Kueichulin Formation; Sample no. 5 is NN13 - 15, equivalent to the upper part of the Chinshui Shale; Samples nos. 6 - 8 are NN15, equivalent to the Chinshui Shale; and Sample no. 9 is NN16 - 18, equivalent to the Cholan Formation. All the results of nannofossil analysis correlate strongly with the geological map of the CPC.

Additionally, in a previous study, related samples from BH1, BH1A, and CLF-2 boreholes fall in the biozone range of NN14 - 15 (Matini's fossil zone, Martini 1971), which is upper Early Pliocene in age and equivalent to the Yutenping Sandstone Member and the Chinshui Shale along the Holung-Chi sections of the Miaoli area, northern Taiwan. The boundary between the Chinshui Shale and the Kueichulin Formation in BH-1 borehole is located at $210.5-225.6 \mathrm{~m}$. This assessment is based on the distribution richness of nannofossil peak variation of the nannofossil content at the $\mathrm{BH}-1 \mathrm{~A}$ borehole and probably originates from the change of parasequence between different delta systems within the Yutenping Sandstone. The boundary between the Chinshui and the Kueichulin Formations is judged by lithofacies and sedimentary facies (Huang et al. 2002). Nannofossil columns from these nearby wells should provide good correlation to those of TCDP well-A.

If we put the nannofossil zones, richness distributions, litho-stratigraphic, and structural features together (Fig. 13) we find that the Chinshui shale corresponds with the richest nannofossil zone, while the lower Cholan Formation is subordinate and the upper Cholan and Kueichulin Formations seem to lack large amounts of nannofossils. The Chinshui Shale is characterized by mudstone, some siltstone and rare fine-grain sandstone. The sedimentary features are dominated by aggradation rather than the progradation in gamma ray and lithologic columns. The paleo-environment of the Chinshui Shale is probably open marine. The overlying Cholan formation is dominated by very fine-grain muddy sandstone and interbed with siltstone and mudstone. Prograding features are highly developed in the lower part of this Cholan Formation (Lin et al. 2007). The underlying formation of the Chinshui Shale is the Kueichulin Formation, which is dominated by the fine-grain clean sandstone interbedded with very fine-grain sandstone and rare siltstone. The clean and coarse-grain sandstone occurs in the upper part of the Kueichulin Formation rather than the lower part. In the depth interval 1710 - $2003 \mathrm{~m}$ of TCDP well-A, the nannofossil zone is determined to be NN16 - 18 or part of the Cholan Formation, which is dominated by very fine-grain sandstone and interbedded with fine-grain sandstone and rare siltstone in lithology. The repetition of the Cholan Formation also determines the depth of the Sanyi Fault. The deep repeating section of the Cholan Formation differs slightly from the shallow Cholan Formation in lithology. Based on the structural interpretation of TCDP well-A and its geological section by Hung et al. (2007), the first drilled section of Cholan is the typical lower Cholan Formation, while the second drilled section is typical of the middle Cholan Formation. Our nannofossil analysis has proposed good criteria to help determine the Sanyi Fault zone within the muddy fine and very fine grain regime. Many Chelungpu Fault zones occur in the Chinshui Shale and the boundary between the Chinshui Shale and the Kueichulin Formation. These Chelungpu shear zones are actually the 
Table 12. Nannofossil analysis from typical outcrop samples near by the Chelungpu fault, Taichung.

\begin{tabular}{|c|c|c|c|c|c|c|c|c|c|}
\hline Outcrop sample number & - & $\sim$ & m & + & in & $\bullet$ & - & $\infty$ & $a$ \\
\hline ABUNDANCE per 10 fields & $?$ & 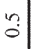 & ง & $\infty$ & r & 2 & $\because$ & $m$ & $a$ \\
\hline \multicolumn{10}{|l|}{ Amaurolithus amplificus } \\
\hline A. delicatus & & $\mathrm{P}$ & & & & & P & & \\
\hline A. primus & & & & & & & & & \\
\hline A. tricorniculatus & & & & & & & & & \\
\hline \multicolumn{10}{|l|}{ Coccolithus pelagicus } \\
\hline \multicolumn{10}{|l|}{ Catinaster calyculus } \\
\hline C. coalitus & & & & & & & & & \\
\hline \multicolumn{10}{|l|}{ Ceratolithus acutus } \\
\hline C. armatus & & & & & & & ... & & ......... \\
\hline C. cristatus & & & & & & & & & \\
\hline C. rugosus & & & & & & & & & \\
\hline \multicolumn{10}{|l|}{ Coronocyclus nitescens } \\
\hline Cyclicargolithus floridanus & & R & & & & $\mathrm{R}$ & R & $\mathrm{R}$ & R" \\
\hline \multicolumn{10}{|l|}{ C. abisectus } \\
\hline Calcidiscus leptoporus & & & & R & & & & & \\
\hline C. macintyrei & & & & $\mathrm{R}$ & & & & & \\
\hline Dictyococcites bisectus & & & & & & & & & \\
\hline D. productus & & & & & & & & & \\
\hline \multicolumn{10}{|l|}{ Discoaster adamanteus } \\
\hline D. asymmetricus & & & & & & & & & \\
\hline D. bellus & & & & & & & $\cdots$ & & \\
\hline D. bollii & & & & & & & $\cdots$ & & \\
\hline D. brouweri & & & & & & & & & \\
\hline D. calcaris & & & & & & & & & \\
\hline D. deflandrei & & & & & & & & & $R$ \\
\hline D. druggi & & & & & & & & & \\
\hline D. exilis & & & & & & & & & ........... \\
\hline D. hamatus & & & & & & & & & \\
\hline D. neohamatus & & & & & & & 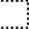 & & \\
\hline D. pentaradiatus & & & & & & & & & \\
\hline D. quinqueramus & & & & & & & & & \\
\hline D. surculus & & & & & & & P. & & \\
\hline D. tamalis & & & & & & & & & $\cdots$ \\
\hline D. variabilis & $\mathrm{p}$ & & & & & & & & \\
\hline \multicolumn{10}{|l|}{ Emiliania huxleyi } \\
\hline \multicolumn{10}{|l|}{ Ericsonia fenestrata } \\
\hline Gephyrocapsa spp. & & & R & & & & $\mathrm{R}$ & & $R$ \\
\hline G. oceanica & & & & & & & & & \\
\hline Helicosphoera ampliaperta & & & & & & & & & \\
\hline H. carteri & & & & & & & & & $R$ \\
\hline H. intermedia & & & & & & & & & \\
\hline H. obliqua & & & & & & & & & \\
\hline H. sellii & p & & $\mathrm{R}$ & & $R$ & & & & \\
\hline Oolithotus fragilis & & & & & & & & & \\
\hline Pseudoemiliania lacunosa & & & & & $P$ & & $\mathrm{R}$ & & \\
\hline Reticulofenestra spp. & P” & & $\mathrm{R}$ & FC: & $\mathrm{R}$ & & R & R" & $R$ \\
\hline R. minutula & & $\mathrm{R}$ & $\mathrm{R}$ & R & $\mathrm{R}$ & & $R$ & & $R$ \\
\hline R. pseudoumbilica & & & & R & & & $\mathrm{R}$ & $\mathrm{R}$ & $R$ \\
\hline Rhabdosphaera clavigera & & & & & & & & & \\
\hline Sphenolithus abies & & & & $\mathrm{R}$ & & & & & \\
\hline S. belemnos & & & & & & & & & \\
\hline S. ciperoensis & & & & & & & & & \\
\hline S. conicus & & & & & & & & & \\
\hline S. dissimilis & & & & & & & & & \\
\hline S. distentus & & & & & & & & & \\
\hline S. heteromorphus & & & & & & & & & \\
\hline S. moriformis & & & & & & & & & \\
\hline S. neoabies & & & & & & & & & \\
\hline Syracosphaera histrica & & & & & & & & & \\
\hline Umbilicosphaera sibogae & & & & & & & & 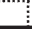 & \\
\hline
\end{tabular}


flexure bedding slip thrust so that it is difficult to find repetitions of the lithologic section or the fossil zones.

On the other hand, Wen-Shan Chen (2006, personal communication) speculated that the age of the interval at 1717.7 - $2003 \mathrm{~m}$ might not be Late Pliocene but rather the same age as that of the Kueichulin Formation. In his opinion, because 1951.5 m contains Discoaster Pentaradiatus (if the fossils are not reworked), a comparison with the fossil assemblages in the upper core shows that the fossils only appear in the Chinshui Shale and the upper Kueichulin Formation. Thus, he also suggests that neither of the faults penetrating 1782 and $1848 \mathrm{~m}$ can

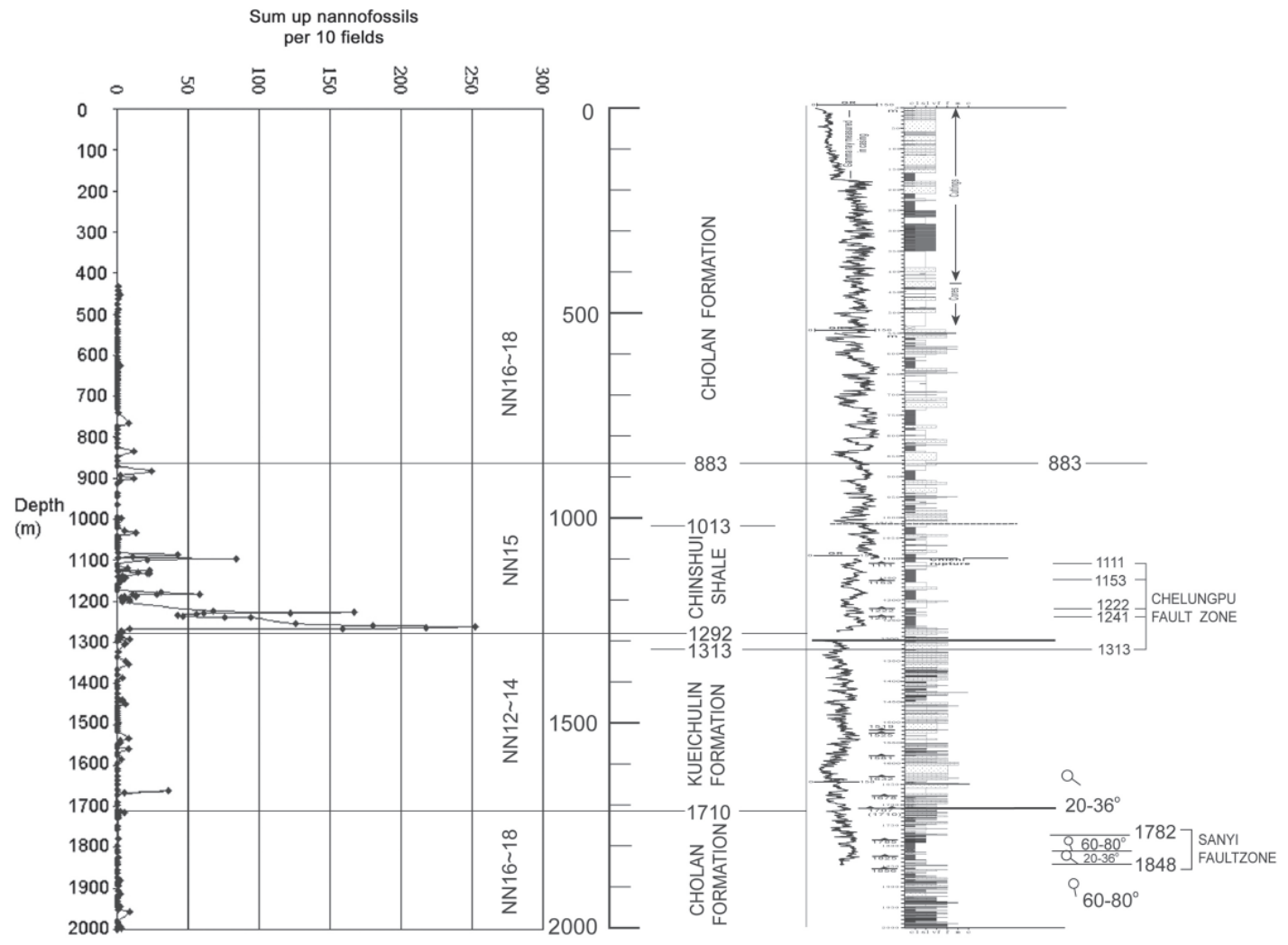

Fig. 13. The nannofossil zoning and richness distribution in relation to the lithostratigraphic (Lin et al. 2007) and structural (Hung et al. 2007) interpretations. Many Chelungpu Fault zones occur within Chinshui Shale (NN15) and the boundary between the Chinshui Shale and Kueichulin Formation (between NN15 and NN12 - 14). Therefore, the Chelungpu Fault zone is better termed a no flexure bedding slip fault and it is difficult to find the fossil zone repetition while the Sanyi Fault determined by the bedding change and the clear repetition of the fossil zone. 


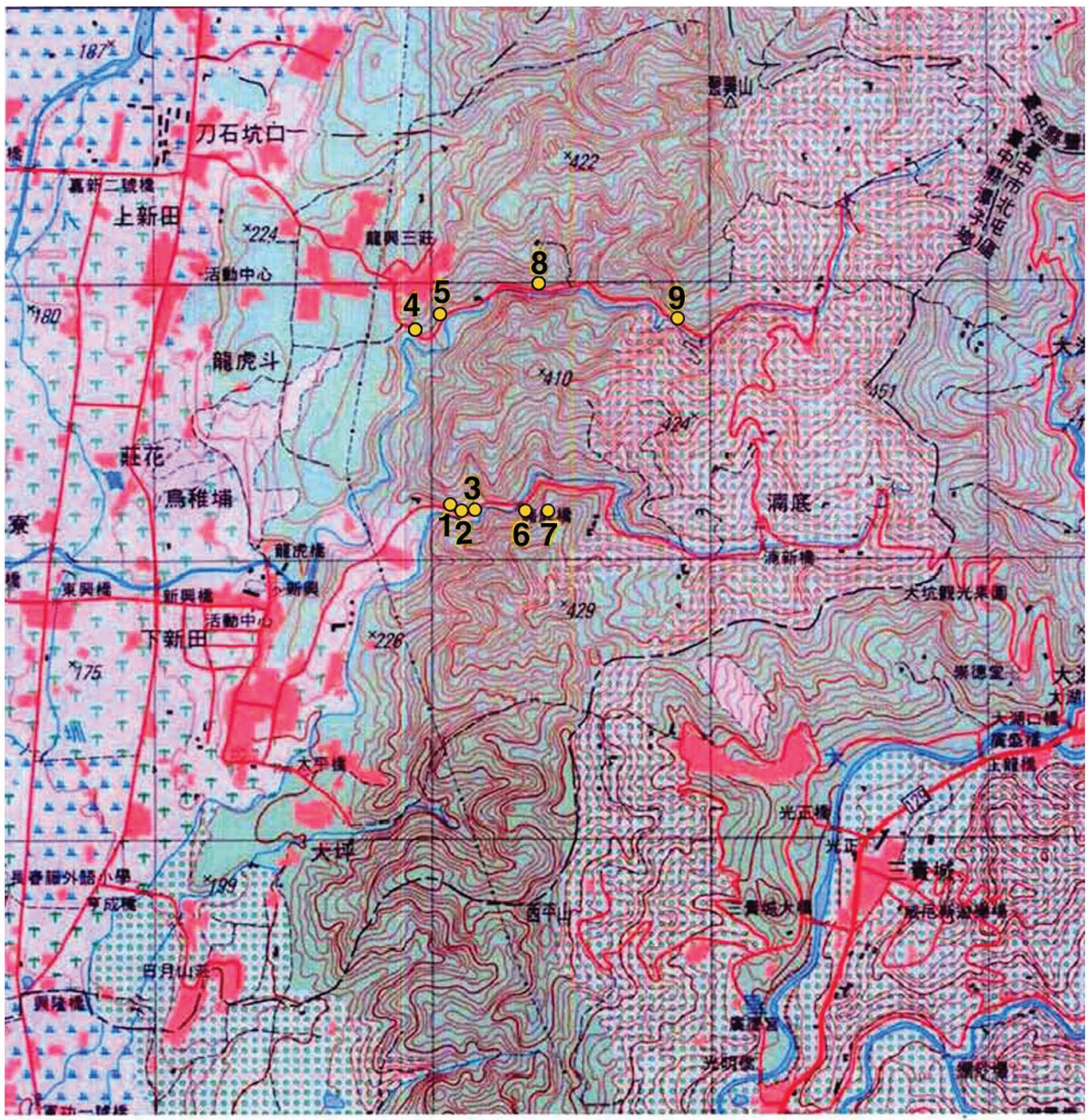

\begin{tabular}{|c|c|c|}
\hline Sample No. & $\mathrm{X}$ & $\mathrm{Y}$ \\
\hline 1 & 222085 & 2678167 \\
\hline 2 & 222137 & 2678157 \\
\hline 3 & 222148 & 2678173 \\
\hline 4 & 221929 & 2678831 \\
\hline 5 & 222019 & 2678873 \\
\hline
\end{tabular}

\begin{tabular}{|c|c|c|}
\hline Sample No. & $\mathrm{X}$ & $\mathrm{Y}$ \\
\hline 6 & 222320 & 2678192 \\
\hline 7 & 222416 & 2678172 \\
\hline 8 & 222394 & 2678975 \\
\hline 9 & 222866 & 2678813 \\
\hline
\end{tabular}

Fig. 14. Location map of the samples for nannofossil analysis. 
be the Sanyi Fault. Nonetheless, these nannofossils assemblages appearing in the interval 1717.7 - $2003 \mathrm{~m}$ are very similar to those reworked assemblages in the Cholan Formation of the shallower part of the drilled well. Since in-situ fossil assemblages are very rare in the Cholan Formation, the characteristics of reworked assemblages is the only criteria for us to identify the appearance of the formation. Therefore, we suggest that the interval be the Cholan Formation and that the top of the repeated formation could be the depth of the penetrating Sanyi fault.

\section{CONCLUSIONS}

The TCDP well-A penetrates the fine-grain muddy sandstone and sandy mudstone formations of the Pliocene and Pleistocene in the Taichung area. The soft cores have difficulty satisfying all the requests of different geo science regimes. Therefore, core preservation by standard procedures and nannofossil analysis to determine formation boundaries has become very important, especially during well drilling. In this study, a 456 meter subsurface core has been coated in resin and slabbed, and core fabrics digitized. More than 150 rock samples have been analyzed for nannofossil to constrain a detailed biostratigraphic column on TCDP well-A. The fossil zones at depth intervals are given as follows: 431 - $883 \mathrm{~m}$ is NN16 - 18; $883-1226 \mathrm{~m}$ is NN12 - 15; 1239 - $1710 \mathrm{~m}$ is NN12 - 14, and the residual bottom interval is NN16 - 18; an interval which is also abundant with reworked fossil assemblages. This study also reveals the repetition of the younger fossil zone NN16 - 18 in the bottom interval of the well and verifies the exact subsurface position of the Sanyi Fault.

Acknowledgements This project "Core Slabbing and Nannofossil Analysis of the Chelungpu Fault Zone" was submitted from the National Central University during February 2004 - June 2005. Authors thank Dr. C. Y. Wang and Dr. K. F. Ma of the National Central University, and Dr. S. R. Song of the National Taiwan University for their kind help. Authors are also very grateful to Dr. K. A. Lin, director of the Exploration and Development Research Institute (EDRI), H. Y. Chen, W. B. Sue, Y. L. Wu, C. J. Hsu, and H. Y. Chang at EDRI for support and help in this project. Authors are also very grateful to Dr. Wen-Shan Chen of Taiwan University and Mr. Kai-Shuan Shea of the Chinese Geological Survey, Taiwan for their revisions and proposing good guidance in the production of this article. This article is a TEC Contribution Number 00009.

\section{REFERENCES}

Chi, W. R., 1981: Nannofossils, Exploration and Development Research Center, 407 pp. (in Chinese)

Huang, S. T., J. C. Wu, J. H. Hung, and H. Tanaka, 2002: Studies of sedimentary facies, stratigraphy, and deformation structures of the Chelungpu fault zone on cores from drilled wells in Fengyuan and Nantou, Central Taiwan, Terr. Atmos. Ocean. Sci., 13, 253-278. 
Huang, T. C., 1982: Tertiary calcareous nannofossil strtigraphy and sedimentation cycles in Taiwan, Proc. $2^{\text {nd }}$ ASCOPE conference and exhibition, 873-886.

Hung, J. H., S. T. Huang, and J. C. Wu, 2001: Lithofacies and biostratigraphy of the core from the boreholes drilled through the Chelungpu fault, ICDP Workshop on Drilling the Chelungpu Fault, San Francisco.

Hung, J. H., and J. Suppe, 2002: Subsurface geometry of the Sani-Chelungpu faults and fold scarp formation in the 1999 Chi-Chi Taiwan earthquake, AGU 2002 Fall Meeting.

Hung, J. H., Y. H. Wu, E. C. Yeh, J. C. Wu, and TCDP Scientific Party, 2007: Subsurface structure, physical properties, and fault zone characteristics in the scientific drill holes of Taiwan Chelungpu-fault Drilling Project. Terr. Atmos. Ocean. Sci., 18, 271-293, doi: 10.3319/TAO.2007.18.2.271(TCDP).

Lin, A. T., S. M. Wang, J. H. Hung, M. S. Wu, and C. S. Liu, 2007: Lithostratigraphy of the Taiwan Chelungpu-fault Drilling Project-A borehole and its neighboring region, central Taiwan. Terr. Atmos. Ocean. Sci., 18, 223-241, doi: 10.3319/TAO.2007.18.2.223 (TCDP).

Martini, E., 1971: Standard tertiary and quaternary calcareous nannoplankton zonation. In: Farinacci, A. (Ed.), Proc. II Plank. Conf. Rome, 1970, 737-785.

Okada, H., and D. Bukry, 1980: Supplementary modification and introduction of code numbers to the low-latitude coccolith biostratigraphic zonation. Mar. Micropaleontol., 5, 321-325.

Wu, J. C., C. T. Tsai, W. W. Mei, M. H. Wang, K. M. Yang, S. T. Huang, M. Lee, W. R. Chi, 2002: Reapproach of Gutingkeng Sequence in the west side of Lungchuan Fault, 2002 Annual meeting of China Geology Society, 7-99. (in Chinese)

Wu, J. C., S. T. Huang, M. H. Wang, C. C. Tsai, W. W. Mei, J. H. Hung, T. Y. Lee, K. M. Yang, and K. F. Lee, 2007: Core slabbing and nannofossil analysis on the Chelungpu fault zone, Taichung, Taiwan. Terr. Atmos. Ocean. Sci., 18, 295-325, doi: 10.3319/ TAO.2007.18.2.295(TCDP). 